\title{
Clinical Efficacy of Qili Qiangxin Capsule Combined with Western Medicine in the Treatment of Chronic Heart Failure: A Systematic Review and Meta-Analysis
}

\author{
Xiaoming Xu, ${ }^{1}$ Yang Yang, ${ }^{2}$ Gendong Zhou, ${ }^{2}$ Zhengxuan Du, ${ }^{2}$ Xiaohong Zhang, ${ }^{2}$ Wei Mao, \\ and Hongwen Cai ${ }^{1}{ }^{1}$ \\ ${ }^{1}$ Department of Cardiology, The First Affiliated Hospital of Zhejiang Chinese Medical University, Hangzhou, Zhejiang, China \\ ${ }^{2}$ Department of Cardiology, The Third Affiliated Hospital of Anhui Medical University, Hefei 230061, Anhui, China \\ Correspondence should be addressed to Hongwen Cai; hwcai_zj0121@126.com
}

Received 18 April 2021; Revised 4 June 2021; Accepted 31 July 2021; Published 9 August 2021

Academic Editor: Mohammad Hashem Hashempur

Copyright (C) 2021 Xiaoming Xu et al. This is an open access article distributed under the Creative Commons Attribution License, which permits unrestricted use, distribution, and reproduction in any medium, provided the original work is properly cited.

\begin{abstract}
Qili Qiangxin capsule (QQC) is a formulation of traditional Chinese medicine commonly used for the treatment of heart failure in China. This meta-analysis aimed to assess the clinical efficacy of QQC combined with western medicine in the treatment of chronic heart failure (CHF). We conducted a systematic review and meta-analysis abided by the PRISMA guidelines. Literature search was conducted in the China National Knowledge Infrastructure, Wanfang Database, Chinese Scientific Journals Database, PubMed, and Web of Science from inception to August 2020. A total of 52 eligible studies were obtained, and 42 of these studies were included in the meta-analysis. The results showed that, compared with western medicine alone, the combination of Qili Qingxin capsule and Western medicine treatment has better efficacy (metoprolol: RR: 1.24, 95\%CI 1.14-1.34; carvedilol: RR: 1.24, 95\%CI 1.14-1.34; trimetazidine: RR: 1.20, 95\%CI: 1.12-1.27; sacubitril valsartan sodium: RR: 1.23, 95\%CI: 1.11-1.36; sodium nitroprusside: RR: 1.33, 95\%CI: 1.23-1.45; and bisoprolol: RR: 1.31, 95\%CI: 1.15-1.49) and increased the level of LVEF, LVEDD, and 6MWT of patients with CHF and reduced the adverse effects and the level of HR, LVESD, BNP, and Hs-cTnT as well. However, there is high heterogeneity in the meta-analysis of LVEDV, BNP, NT-proBNP, Hs-cTnT, 6MWT, and adverse effects, and the methodological quality of the included studies was poor. Therefore, further studies with good methodological quality and large sample size are required to validate our findings. In our study, evidence suggests that Qili Qiangxin capsule combined with Western medicine may improve therapeutic effect and the quality of life of patients with CHF.
\end{abstract}

\section{Introduction}

Chronic heart failure (CHF) has been recognized as one of the major global health issue which is associated with high risk of morbidity, mortality, and cost [1]. According to the survey, there are 23 million patients with CHF worldwide, approximately 5.7 million in the US and over 4.2 million in China [1]. The pathogenesis of CHF mainly includes primary myocardial damage resulted from long-term glucose and lipid metabolism disorder and ischemic myocardial damage [2]. Especially, the elderly showed higher morbidity of CHF [3]. In the future, the number of patients with CHF would increase significantly with the aging of the population [4].
Conventional Western medicine for CHF treatment includes beta-blockers, cardiotonic steroids, diuretics, and angiotensin-converting enzyme inhibitors (ACEI). Although these treatments have certain therapeutic effects, such as a reduction of heart rate and left ventricular filling pressure and an improvement in exercise capacity [4], an improvement in the curative effect is still necessary because of the adverse effects and short-term effects. For example, the metoprolol, a beta-blocker, may cause depression and anxiety during the CHF treatment [5]. With the development of science and technology, more and more antiCHF ingredients have also been discovered [6-8]. Complementary and alternative medicine therapies have 
become increasingly popular and are used regularly by patients with chronic disorders [9]. Currently, the combination of traditional Chinese medicine (TCM) and western medicine has been widely used for the CHF treatment [10-12]. According to the perspective of TCM, the etiology of CHF is the yang deficiency of heart which is the result of the inadequacy of Qi and blood, blood stasis, and phlegm-dampness. With the in-depth research of TCM, the main CHF treatment is replenishing Qi and nourishing Yin [13].

Qili Qiangxin capsule (QQC) is a traditional Chinese herbal remedy that was mentioned as a treatment with certain therapeutic effects, such as reducing heart rate and revering ventricular remodeling, for the CHF by China Food and Drug Administration [14]. QQC is a standardized Chinese herbal extract prepared from 11 Chinese herbs, including dry root of Astragalus membranaceus (Fisch.) Bge. var. mongholicus (Bge.), Hsiao or Astragalus membranaceus (Fisch.) Bge., dry root of Panax ginseng C. A. Meyer, Aconitum carmichaeli Debx., dry root of Salvia miltiorrhiza Bunge, dry seeds of Lepidium apetalum Willd. or Descurainia Sophia (L.) Webb ex prantl., dry tubers of Alisma orientalis (Sam.) Juzep., dry rhizome of Polygonatum odoratum (Mill.) Druce, dry twigs of Cinnamomum cassia Presl, dry flower of Carthamus tinctorius L., dry root bark of Periploca sepium Bge., and dried ripe peel of Citrus reticulata Blanco. Previous studies have reported that QQC played a role in the treatment of CHF through multiple mechanisms, such as reducing cardiac fibrosis remolding, improving cardiac function, reducing NT-proBNP, and regulating the inflammatory responses [13-16]. In addition, the efficacy and safety also have been proved in a study of the effects of QQC on 512 patients with CHF [17].

Currently, QQC combined with Western medicine has been widely used for the treatment of CHF. A lot of studies have reported that QQC combined with Western medicine can improve clinical outcomes compared to Western medicine treatment [18-20]. Sun et al. performed a metaanalysis in 2016 and concluded that QQC plus conventional treatment showed better efficiency than conventional treatment alone in the treatment of heart failure [21]. However, a subgroup analysis based on conventional treatments was not conducted in the meta-analysis. With the deepening of research on QQC in CHF in recent years, it is necessary to conduct an in-depth analysis of the clinical effects of QQC plus Western medicine on CHF patients. Given the small sample sizes and inconsistent results of previous research studies, this study aimed at conducting a systematic review and meta-analysis of the efficacy and safety of QQC combined with Western medicine in the treatment of $\mathrm{CHF}$ and providing reference for clinical diagnosis and treatment.

\section{Method}

This study was compiled based on the Preferred Reporting Items for Systematic reviews and Meta-Analyses statement (PRISMA) (Supplementary Materials S1) [22].
2.1. Search Strategy. We searched the following five databases from their start date to August 2020: China National Knowledge Infrastructure (CNKI), Wanfang Database, Chinese Scientific Journals Database (VIP), PubMed, and Web of Science. We used the following keywords and medical subject heading terms: ("Qili Qiangxin" OR "qiliqiangxin" OR "qiangxinli") and ("Chronic heart failure" OR "Chronic cardiac failure" OR "Chronic heart decompensation"). We also hand-searched the reference lists of all full text papers for additional relevant reports. We did not impose any language restrictions.

2.2. Eligibility and Exclusion Criteria. Trials were considered eligible if they were (a) randomized controlled trials (RCTs); (b) enrolled participants with CHF; (c) compared the curative effects of the combination of QQC and Western drugs with Western drugs alone. The following studies were excluded, including repeated publication, literature with incomplete or incorrect data, and the control group without a description of the Western drugs used in this study.

2.3. Study Selection. At first, the duplicates of all records obtained from the electronic databases were removed. Then, two researchers read the titles and abstracts independently to identify the eligible studies. Full texts of the studies were read to determine whether they met the eligibility criteria. The disagreements were resolved by discussing with a third researcher.

2.4. Outcomes. Our primary outcome measures were the clinical efficiency. Secondary outcomes measures were heart rate (HR), left ventricular ejection fractions (LVEF), left ventricular end-diastolic dimension (LVEDD), left ventricular end-diastolic volume (LVEDV), left ventricular endsystolic diameter (LVESD), brain natriuretic peptide (BNP), N-terminal Pro B-type natriuretic peptide (NT-proBNP), high-sensitivity cardiac troponin $\mathrm{T}$ (Hs-cTnT), 6-min walk test (6MWT). We also collected adverse events data. RCTs reporting one or more of these outcomes were included.

2.5. Data Extraction and Quality Assessment. The following details were extracted by two researchers independently from included studies: first author; year; sample size; age; gender; course of treatment; the intervention of experimental group; the intervention of control group; and outcome data. The methodological quality of the included studies was used Cochrane 5.1.0 assessment tool. It includes following seven aspects: (1) random sequence generation; (2) allocation concealment; (3) blinding of participants and personnel; (4) blinding of outcome assessment; (5) incomplete outcome data; (6) selective reporting; and (7) other bias. Each domain of the included studies was evaluated as having a low, high, or unclear risk of bias. It was also independently completed by two researchers. The disagreements were resolved by discussing with a third researcher. 
2.6. Data Analysis. The meta-analysis was performed by using RevMan 5.3 software. The relative risk (RR) and 95\% confidence intervals (95\% CI) were used for dichotomous variables, and the mean difference (MD) and 95\% CI were used for continuous variables. This study used $I^{2}$ and $Q$ tests to evaluate heterogeneity of study. When the $I^{2}>50 \%$ or $P<0.01$ in the $Q$ test, the random model would be used for meta-analysis. Otherwise, we used fixed model for metaanalysis.

\section{Results}

3.1. Description of Studies. As shown in Figure 1, a total of 1623 studies were obtained from five databases. After removing the 552 duplicates, 281 mechanism studies, 268 animal experiments, 298 reviews, protocols, and case reports, we included 224 studies. By screening the full text articles, we removed 67 studies of no control group, 48 studies of incomplete or incorrect data, 57 studies of the control group without a description of the Western drugs used in study. Finally, we included 52 studies for the quality assessment, and 42 of these studies were included for metaanalysis.

3.2. Characteristics of Study. The characteristics of the 52 included studies are shown in Table 1. All included studies were conducted in China and published in Chinese. Among the included studies, 11 studies [18-20, 37, 40, 45, 46, 57-60] were related to clinical efficacy of QQC combined with metoprolol. 3 studies [36, 42, 53] were related to clinical efficacy of QQC combined with bisoprolol. 3 studies $[51,69,70]$ were related to clinical efficacy of QQC combined with levocarnitine oral solution. 5 studies [31, 43, 44, 55, 56] were related to clinical efficacy of QQC combined with carvedilol. 10 studies $[24,26,27,32-35,47-49]$ were related to clinical efficacy of QQC combined with trimetazidine. 1 study [23] was related to clinical efficacy of QQC combined with digoxin. 2 studies $[54,64]$ were related to clinical efficacy of QQC combined with coenzyme Q10. 1 study [65] was related to clinical efficacy of QQC combined with enalapril maleate and folic acid tablets. 4 studies $[61,66-68]$ were related to clinical efficacy of QQC combined with sacubitril valsartan sodium tablets. 1 study [30] was related to clinical efficacy of QQC combined with benazepril hydrochloride. 9 studies $[25,28,29,38,39,41,52,63,71]$ were related to clinical efficacy of QQC combined with sodium nitroprusside, and 2 studies [50, 62] were elated to clinical efficacy of QQC combined with ivabradine.

3.3. Risk of Bias. As shown in Figure 2, for the random sequence generation, 6 studies were high risk of bias, and the rest were low risk of bias. One study specified the methods of allocation, and the rest were unclear. One study blinded participants, personnel, and outcome assessment, whereas the rest had no description about the methods of the blinding. There were complete data in all studies, and all studies had low risk of bias in selective reporting and other source of bias.
3.4. Clinical Efficacy. In Figures 3 and 4, the meta-analysis results showed that the clinical efficacy of QQC plus Western drugs treatment was significantly better than Western drugs treatment alone. The following data showed the metaanalysis of QQC combined with different Western drugs treatment: (1) metoprolol ( $n=9$ trials, RR: $1.21,95 \% \mathrm{CI}: 1.14$ to 1.29 , Figure $3(\mathrm{a})$ ); (2) carvedilol ( $n=5$ trials, RR: $1.24,95 \%$ CI 1.14 to 1.34 , Figure 3(b)); (3) trimetazidine ( $n=8$ trials, RR: $1.20,95 \%$ CI: 1.12 to 1.27 , Figure $3(\mathrm{c})$ ); (4) sacubitril valsartan sodium ( $n=4$ trials, RR: 1.23 , 95\%CI: 1.11 to 1.36 , Figure 4(a)); (5) sodium nitroprusside ( $n=8$ trials, RR: 1.33 , 95\%CI: 1.23 to 1.45 , Figure 4(b)); and (6) bisoprolol $(n=3$ trials, RR: $1.31,95 \% \mathrm{CI}: 1.15$ to 1.49 , Figure $4(\mathrm{c})$ ).

3.5. Heart Rate (HR). The meta-analysis with a random model showed that the HR of QQC plus metoprolol treatment was significantly lower than metoprolol treatment alone $(n=8$ trials, $\mathrm{MD}:-8.71,95 \% \mathrm{CI}:-10.93$ to $-6.50, P<0.00001$, heterogeneity $\chi^{2}=66.92, P<0.00001, \mathrm{I}^{2}=90$, Figure 5).

3.6. Left Ventricular Ejection Fractions (LVEF). The metaanalysis results showed that the LVEF of QQC plus Western drugs treatment was significantly higher than Western drugs treatment alone (Figures 6 and 7). The following data showed the meta-analysis of QQC combined with different Western drugs treatment: (1) metoprolol ( $n=7$ trials, MD: 3.86, 95\%CI: 2.92 to 4.80 , Figure 6(a)); (2) carvedilol ( $n=5$ trials, MD: 11.02 , 95\%CI: 6.68 to 15.36 , Figure 6(b)); (3) trimetazidine ( $n=8$ trials, MD: $8.08,95 \% \mathrm{CI}: 4.99$ to 11.17 , Figure 6(c)); (4) sacubitril valsartan sodium ( $n=4$ trials, MD: 6.78 , 95\%CI: 4.53 to 9.04, Figure 7(a)); (5) sodium nitroprusside ( $n=6$ trials, MD: $4.37,95 \% \mathrm{CI}$ : 3.33 to 5.40 , Figure 7(b)).

\subsection{Left Ventricular End-Diastolic Dimension (LVEDD).} The meta-analysis results showed that the LVEDD of QQC plus Western drugs treatment was significantly higher than Western drugs treatment alone (Figure 8). The following data showed the meta-analysis of QQC combined with different Western drugs treatment: (1) metoprolol $(n=3$ trials, MD: $-2.98,95 \% \mathrm{CI}:-4.21$ to -1.75 , Figure 8 (a)); (2) carvedilol ( $n=3$ trials, MD: $-7.51,95 \% \mathrm{CI}:-9.85$ to -5.81 , Figure $8(\mathrm{~b})$ ); (3) trimetazidine ( $n=5$ trials, MD: $-4.61,95 \%$ CI: -7.26 to -1.07 , Figure $8(\mathrm{c}))$; (4) sodium nitroprusside ( $n=5$ trials, MD: -5.72 , 95\%CI: -6.95 to -4.50 , Figure $8(d)$ ).

3.8. Left Ventricular End-Diastolic Volume (LVEDV). The meta-analysis with a random model showed that the LVEDV of QQC plus trimetazidine treatment was significantly lower than trimetazidine treatment alone $(n=3$ trials, MD: -25.19 , 95\%CI: -37.69 to $-12.68, P<0.0001$, heterogeneity $\chi^{2}=14.99$, $P=0.0006, I^{2}=87 \%$, Figure 9).

3.9. Left Ventricular End-Systolic Diameter (LVESD). The meta-analysis with a random model showed that the LVESD of QQC plus sodium nitroprusside treatment was 


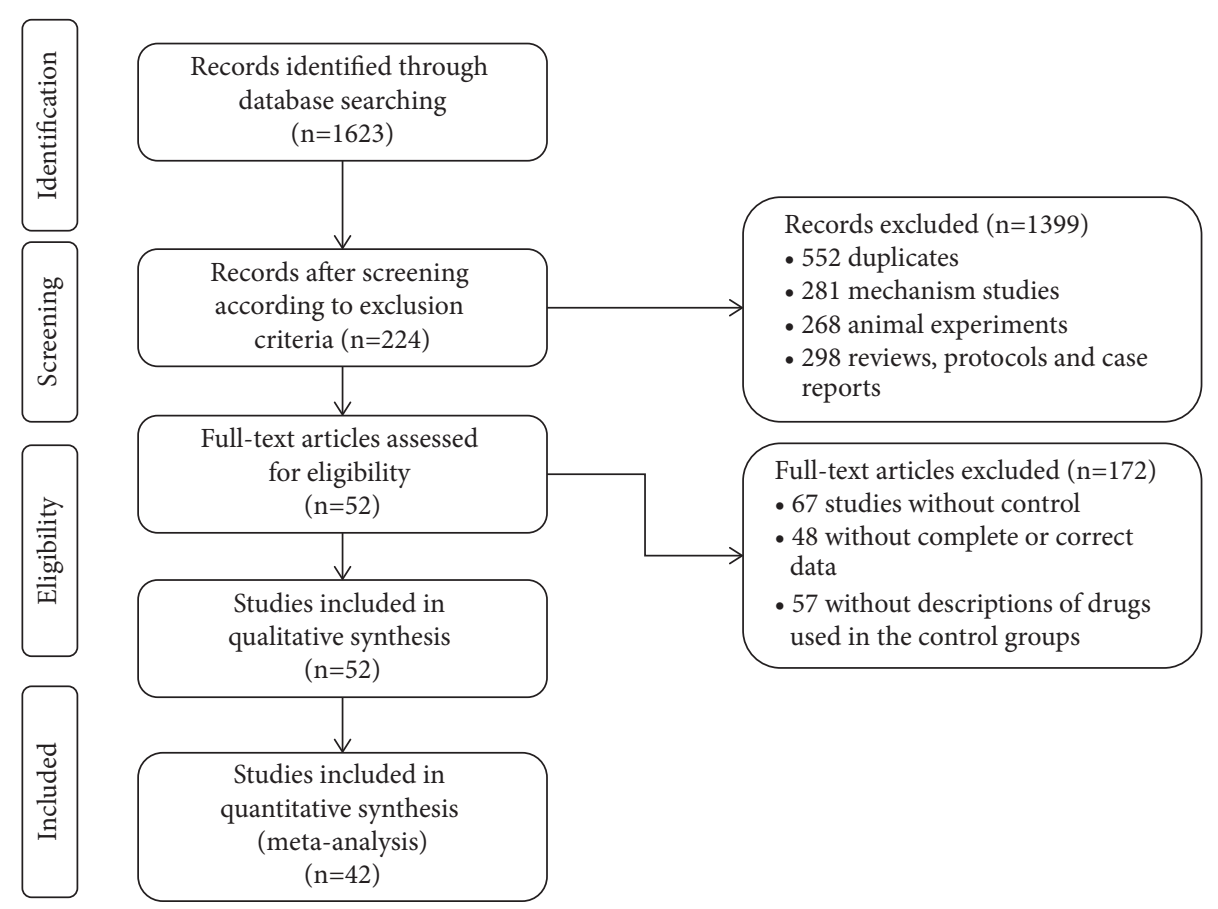

FIGURE 1: Flow chart of study searching and selection.

significantly lower than sodium nitroprusside treatment alone $(n=4$ trials, MD: $-5.64,95 \% \mathrm{CI}:-6.75$ to -4.53 , $P<0.0001$, heterogeneity $\chi^{2}=0.87, \quad P=0.83, \quad I^{2}=0 \%$, Figure 10).

3.10. Brain Natriuretic Peptide (BNP). The meta-analysis results showed that the BNP of QQC plus Western drugs treatment was significantly lower than Western drugs treatment alone (Figure 11). The following data showed the meta-analysis of QQC combined with different Western drugs treatment: (1) metoprolol ( $n=8$ trials, MD: -388.94 , 95\%CI: -488.23 to -289.64 , Figure $11(\mathrm{a})$ ) and (2) bisoprolol $(n=3$ trials, MD: -242.41 , 95\%CI: -428.59 to -56.24 , Figure 11(b)).

3.11. N-Terminal Pro-B-Type Natriuretic Peptide (NTProBNP). The meta-analysis results showed that there was no significant difference between the NT-proBNP of QQC plus Western drugs treatment and Western drugs treatment alone (Figure 12). The following data showed the metaanalysis of QQC combined with different Western drugs treatment: (1) trimetazidine ( $n=3$ trials, MD: $-0.47,95 \% \mathrm{CI}$ : -1.01 to 0.07 , Figure $12(\mathrm{a})$ ) and (2) sacubitril valsartan sodium ( $n=3$ trials, MD: $-0.61,95 \% \mathrm{CI}:-1.38$ to 0.15 , $P=0.12$, heterogeneity $\chi^{2}=171.01$, Figure $12(\mathrm{~b})$ ).

3.12. High-Sensitivity Cardiac Troponin $T$ (Hs-cTnT). The meta-analysis with a random model showed that the HscTnT of QQC plus metoprolol treatment was significantly lower than metoprolol treatment alone $(n=3$ trials, MD: $-5.93,95 \% \mathrm{CI}$ : -9.92 to $-1.95, P=0.004$, heterogeneity $\chi^{2}=69.45, P<0.0001, I^{2}=97 \%$, Figure 13).
3.13. 6-Min Walk Test (6MWT). The meta-analysis with a random model showed that the 6MWT of QQC plus trimetazidine treatment was higher than trimetazidine treatment alone ( $n=3$ trials, MD: $37.61,95 \% \mathrm{CI}: 10.93$ to 64.29 , $P=0.006$, heterogeneity $\chi^{2}=15.94, P=0.0003, I^{2}=87 \%$, Figure 14).

3.14. Adverse Effects. The adverse effects occurred in the included studies included headache, hypotension, hyponatremia, nausea, cough, arrhythmia, and fatigue. The metaanalysis with a random model showed that the adverse events of QQC plus metoprolol treatment was lower than metoprolol treatment alone $(n=8$ trials, RR: $0.48,95 \% \mathrm{CI}$ : 0.28 to $0.81, P=0.006$, heterogeneity $\chi^{2}=16.47, P=0.02$, $I^{2}=58 \%$, Figure $\left.15(\mathrm{a})\right)$. There was no significant differences between the group treated with QQC plus trimetazidine and the group treated with trimetazidine alone $(n=3$ trials, RR: $0.83,95 \% \mathrm{CI}: 0.26$ to $2.67, P=0.76$, heterogeneity $\chi^{2}=0.77$, $P=0.68, I^{2}=0 \%$, Figure $\left.15(\mathrm{~b})\right)$.

\section{Discussion}

The characteristics of CHF are high risk of hospitalization, readmissions, mortality, morbidity, and cost. Patients with CHF suffer from a low quality of life with dyspnea, fatigue, physical exertion, mood disorders, and so on [72]. In recent years, the angiotensin-converting enzyme inhibitors, betablockers, aldosterone antagonists, digoxin, and diuretics as standard Western medicine have been widely used for the treatment of CHF to delay the development of myocardial remodeling [10]. However, owing to the adverse reaction, poor compliance, lower heart rate, and so on, the desired application of these drugs is restricted [2]. The combination 


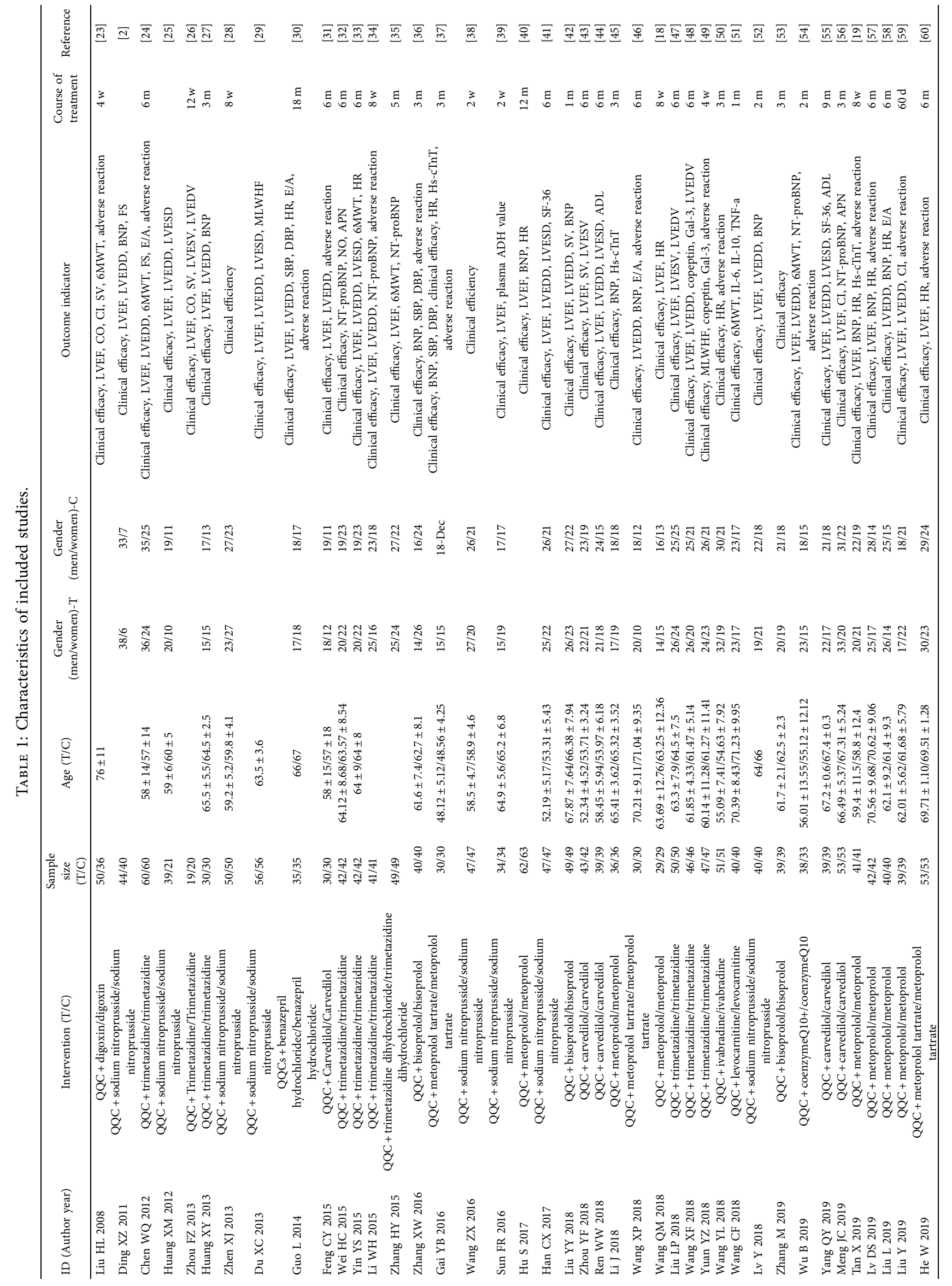




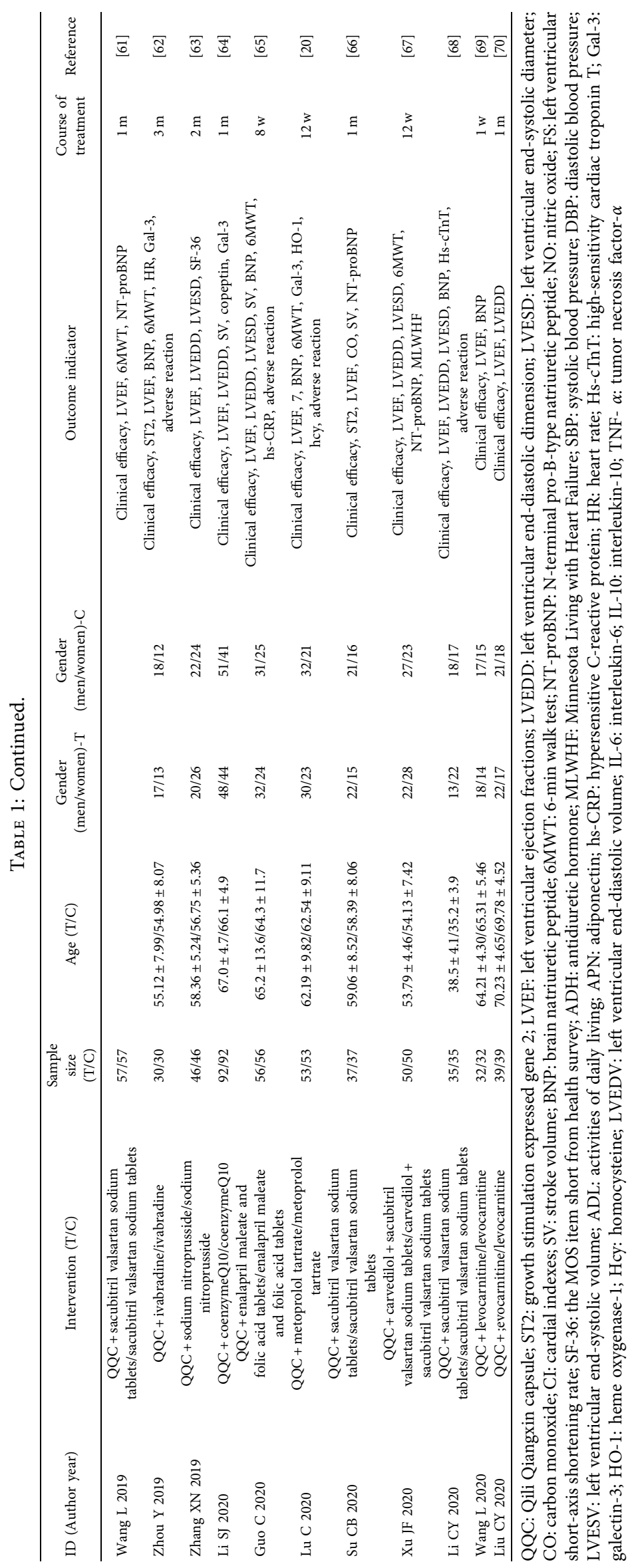



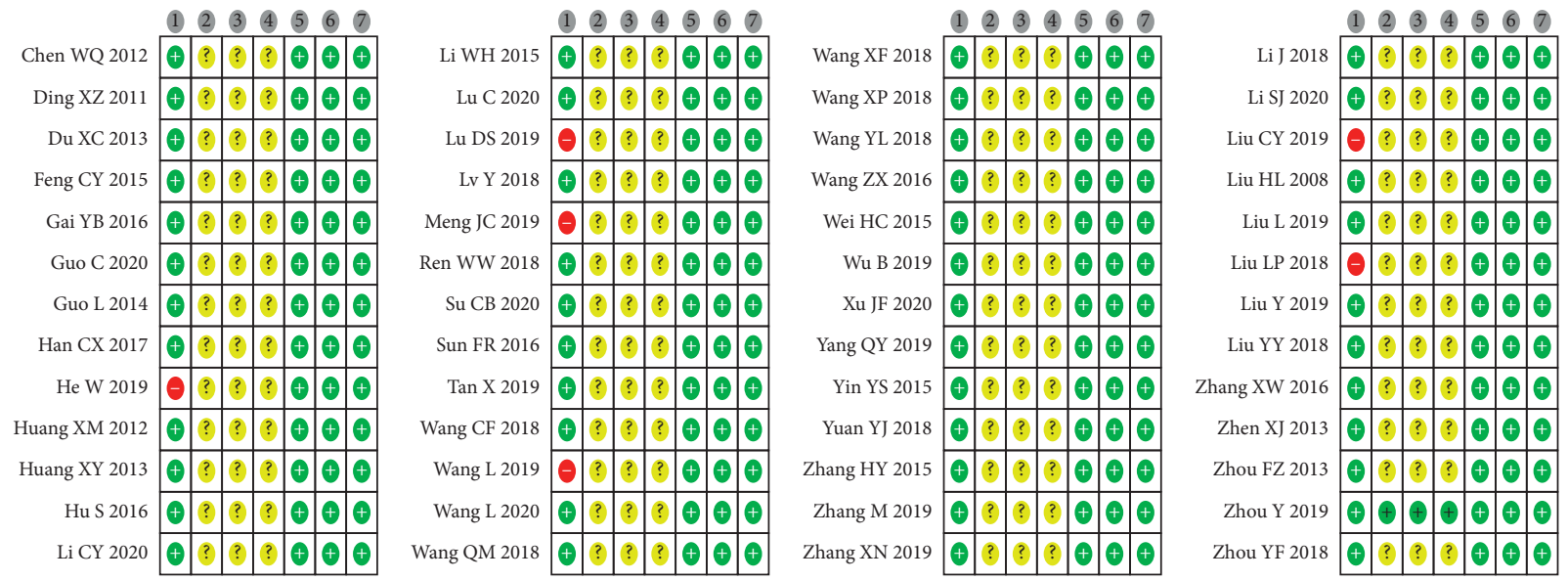

(1) Random sequence generation (selection bias)
4 Blinding of outcome assessment (detection bias)

2 Allocation concealment (selection bias)

Blinding of participants and personnel (performance bias)

FIGURE 2: Risk of bias summary.

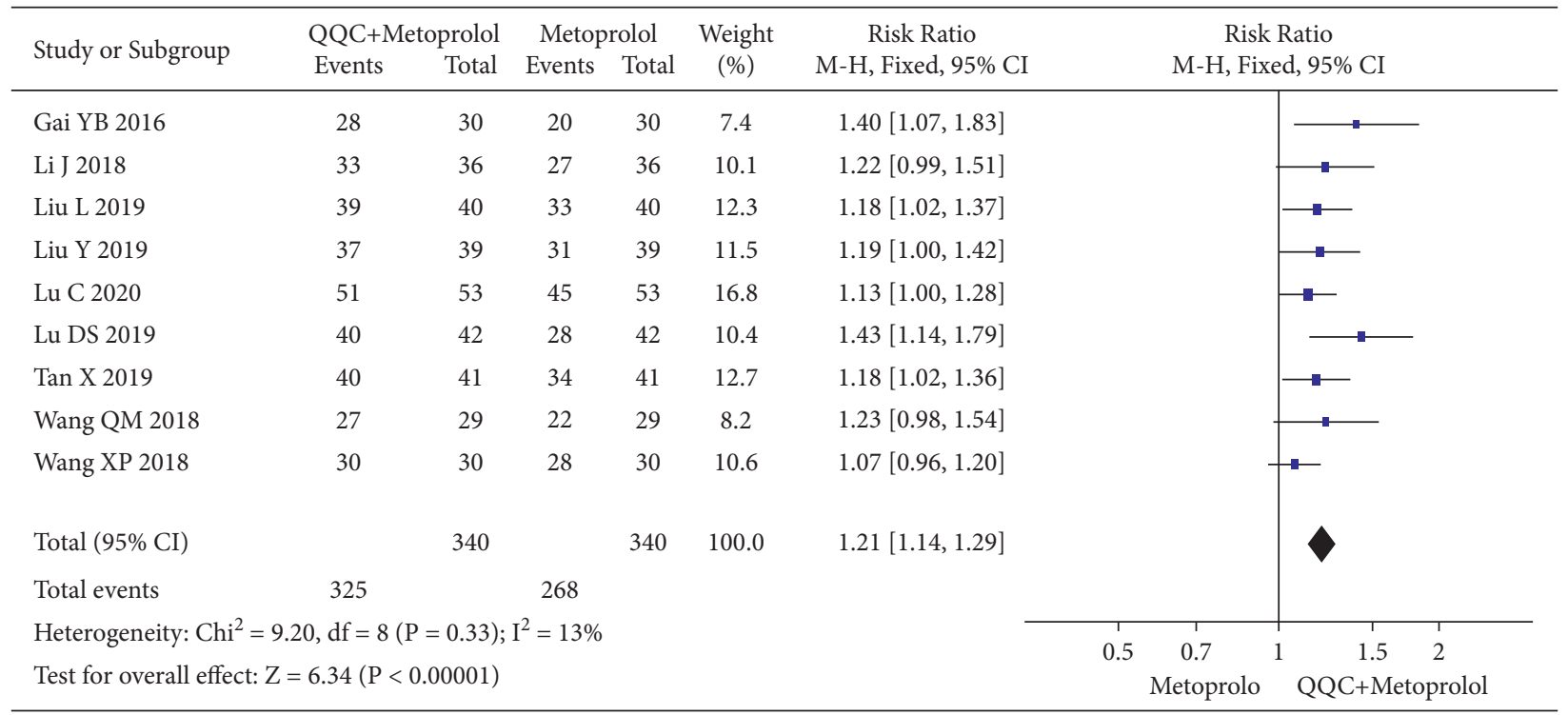

(a)

\begin{tabular}{|c|c|c|c|c|c|c|c|c|c|c|}
\hline \multirow{3}{*}{$\begin{array}{l}\text { Study or Subgroup } \\
\text { Feng CY } 2015\end{array}$} & \multicolumn{2}{|c|}{ QQC+Carvedilol } & \multicolumn{2}{|c|}{ Carvedilol } & \multirow{3}{*}{$\begin{array}{c}\text { Weight } \\
(\%)\end{array}$} & \multirow{3}{*}{$\begin{array}{c}\text { Risk Ratio } \\
\text { M-H, Fixed, 95\% CI } \\
1.33[1.04,1.72]\end{array}$} & \multirow{2}{*}{\multicolumn{3}{|c|}{$\begin{array}{c}\text { Risk Ratio } \\
\text { M-H, Fixed, 95\% CI }\end{array}$}} & \\
\hline & \multirow{2}{*}{$\begin{array}{c}\text { Events } \\
28\end{array}$} & \multirow{2}{*}{$\begin{array}{c}\text { Total } \\
30\end{array}$} & \multirow{2}{*}{$\begin{array}{c}\text { Events } \\
21\end{array}$} & \multirow{2}{*}{$\begin{array}{c}\text { Total } \\
30\end{array}$} & & & & & & \\
\hline & & & & & & & & & - & \\
\hline Meng JC 2019 & 50 & 53 & 42 & 53 & 26.9 & $1.19[1.02,1.39]$ & & & - & \\
\hline Ren WW 2018 & 37 & 39 & 28 & 39 & 17.9 & $1.32[1.07,1.63]$ & & & $\longrightarrow$ & \\
\hline Yang QY 2019 & 38 & 39 & 34 & 39 & 21.7 & $1.12[0.98,1.27]$ & & & 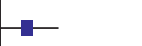 & \\
\hline Zhou YF 2018 & 41 & 43 & 31 & 42 & 20.1 & $1.29[1.07,1.57]$ & & & $\longrightarrow$ & \\
\hline Total $(95 \% \mathrm{CI})$ & & 204 & & 203 & 100.0 & $1.24[1.14,1.34]$ & & & & \\
\hline Total events & 194 & & 156 & & & & & & & \\
\hline Heterogeneity: $\mathrm{Chi}^{2}$ & $\mathrm{df}=4$ & $0.48)$ & $=0 \%$ & & & & 0.5 & 0.7 & 1.5 & 2 \\
\hline Test for overall effec & $5.13(\mathrm{P}$ & $0001)$ & & & & & & Carvedilol & QQC+Car & vedilol \\
\hline
\end{tabular}

(b)

Figure 3: Continued. 


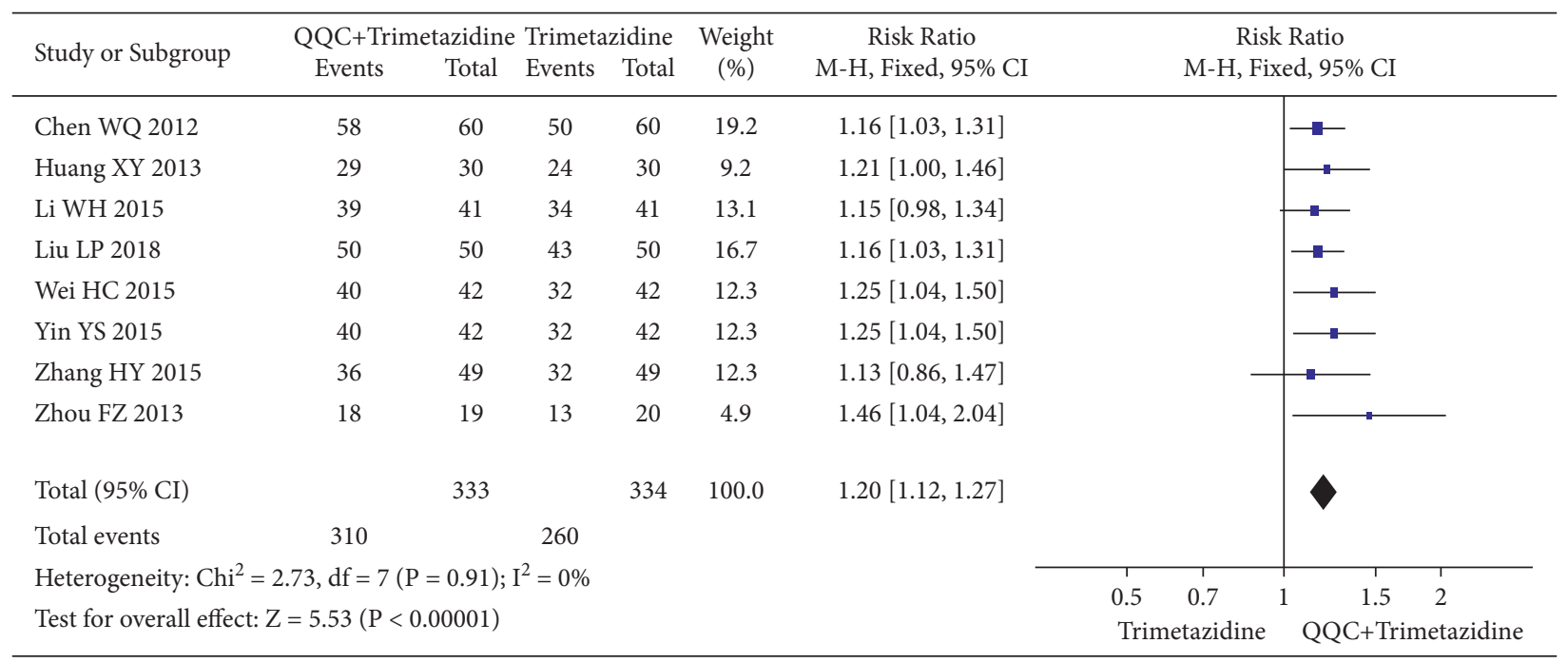

(c)

FIgURE 3: Forest plot of QQC plus Western medicine treatment versus Western medicine only for clinical efficiency (part 1). QQC: Qili Qiangxin capsule.

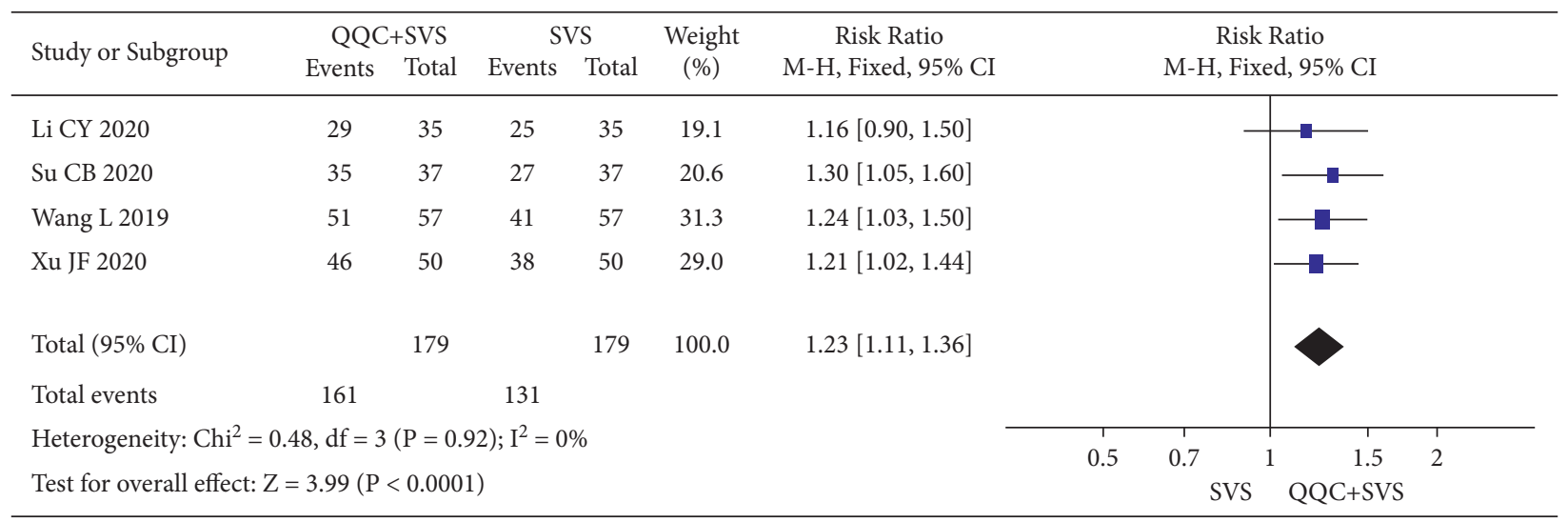

(a)

\begin{tabular}{|c|c|c|c|c|c|c|c|c|c|c|}
\hline \multirow{3}{*}{$\begin{array}{l}\text { Study or Subgroup } \\
\text { Ding XZ } 2011\end{array}$} & \multicolumn{2}{|c|}{$\mathrm{QQC}+\mathrm{SN}$} & \multicolumn{2}{|c|}{ SN } & \multirow{3}{*}{$\begin{array}{c}\begin{array}{c}\text { Weight } \\
(\%)\end{array} \\
11.7\end{array}$} & \multirow{3}{*}{$\begin{array}{c}\text { Risk Ratio } \\
\text { M-H, Fixed, 95\% CI } \\
1.33[1.03,1.72]\end{array}$} & \multirow{2}{*}{\multicolumn{3}{|c|}{$\begin{array}{c}\text { Risk Ratio } \\
\text { M-H, Fixed, 95\% CI }\end{array}$}} & \\
\hline & \multirow{2}{*}{$\begin{array}{c}\text { Events } \\
38\end{array}$} & \multirow{2}{*}{$\begin{array}{c}\text { Total } \\
44\end{array}$} & \multirow{2}{*}{$\begin{array}{c}\text { Events } \\
26\end{array}$} & \multirow{2}{*}{$\begin{array}{c}\text { Total } \\
40\end{array}$} & & & & & & \\
\hline & & & & & & & & & - & \\
\hline Du XC 2013 & 49 & 56 & 32 & 56 & 13.7 & $1.53[1.20,1.96]$ & & & 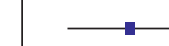 & \\
\hline Han CX 2017 & 40 & 47 & 30 & 47 & 12.9 & $1.33[1.04,1.71]$ & & & $\longrightarrow$ & \\
\hline Huang XM 2012 & 26 & 30 & 18 & 30 & 7.7 & $1.44[1.04,2.00]$ & & & 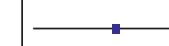 & \\
\hline Sun FR 2016 & 32 & 34 & 25 & 34 & 10.7 & $1.28[1.03,1.59]$ & & & 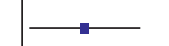 & \\
\hline Wang ZX 2016 & 43 & 47 & 35 & 47 & 15.0 & $1.23[1.02,1.48]$ & & & $\longrightarrow$ & \\
\hline Zhang XN 2019 & 39 & 46 & 30 & 46 & 12.9 & $1.30[1.02,1.66]$ & & & & \\
\hline Zhen XJ 2013 & 46 & 50 & 36 & 50 & 15.4 & $1.28[1.06,1.55]$ & & & $\longrightarrow$ & \\
\hline Total $(95 \%$ CI $)$ & & 354 & & 350 & 100.0 & $1.33[1.23,1.45]$ & & & & \\
\hline Total events & 313 & & 232 & & & & & & & \\
\hline Heterogeneity: Chi & $53, \mathrm{df}=7$ & $(\mathrm{P}=0.9$ & 2); $\mathrm{I}^{2}=0$ & & & & 0.5 & 0.7 & 1.5 & 2 \\
\hline Test for overall effec & $=6.78(\mathrm{P}$ & 0.0000 & & & & & 0.3 & 0.7 & QQC+SN & 2 \\
\hline
\end{tabular}

(b)

Figure 4: Continued. 


\begin{tabular}{|c|c|c|c|c|c|c|c|c|c|c|}
\hline \multirow{3}{*}{$\begin{array}{l}\text { Study or Subgroup } \\
\text { Liu YY } 2018\end{array}$} & \multicolumn{2}{|c|}{ QQC+Bisoprolol } & \multicolumn{2}{|c|}{ Bisoprolol } & \multirow{3}{*}{$\begin{array}{c}\text { Weight } \\
(\%)\end{array}$} & \multirow{3}{*}{$\begin{array}{c}\text { Risk Ratio } \\
\text { M-H, Fixed, 95\% CI } \\
1.21[1.02,1.43]\end{array}$} & \multirow{2}{*}{\multicolumn{3}{|c|}{$\begin{array}{c}\text { Risk Ratio } \\
\text { M-H, Fixed, 95\% CI }\end{array}$}} & \\
\hline & \multirow{2}{*}{$\begin{array}{c}\text { Events } \\
46\end{array}$} & \multirow{2}{*}{$\begin{array}{c}\text { Total } \\
49\end{array}$} & \multirow{2}{*}{$\begin{array}{c}\text { Events } \\
38\end{array}$} & \multirow{2}{*}{$\begin{array}{c}\text { Total } \\
49\end{array}$} & & & & & & \\
\hline & & & & & & & & & - & \\
\hline Zhang M 2019 & 34 & 39 & 25 & 39 & 28.4 & $1.36[1.04,1.77]$ & & & 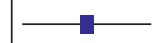 & \\
\hline Zhang XW 2016 & 35 & 40 & 25 & 40 & 28.4 & $1.40[1.07,1.83]$ & & & 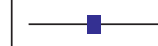 & \\
\hline Total $(95 \%$ CI $)$ & & 128 & & 128 & 100.0 & $1.31[1.15,1.49]$ & & & & \\
\hline Total events & 115 & & 88 & & & & & & & \\
\hline Heterogeneity: $\mathrm{Chi}^{2}$ & $.15, \mathrm{df}=2$ & $(\mathrm{P}=0.5$ & 6); $I^{2}=$ & & & & 0.5 & 0.7 & 1.5 & 2 \\
\hline Test for overall effec & $=4.04(\mathrm{P}$ & $<0.0001)$ & & & & & & Bisoprolol & QQC+Biso & prolol \\
\hline
\end{tabular}

(c)

FIGURe 4: Forest plot of QQC plus Western medicine treatment versus Western medicine only for clinical efficiency (part 2). QQC: Qili Qiangxin capsule, SVS: aacubitril valsartan sodium, and SN: sodium nitroprusside.

\begin{tabular}{|c|c|c|c|c|c|c|c|c|c|c|c|}
\hline \multirow{3}{*}{$\begin{array}{l}\text { Study or Subgroup } \\
\text { Gai YB } 2016\end{array}$} & \multicolumn{3}{|c|}{ QQC+Metoprolol } & \multicolumn{3}{|c|}{ Metoprolol } & \multirow{3}{*}{$\begin{array}{c}\text { Weight } \\
(\%)\end{array}$} & \multirow{3}{*}{$\begin{array}{c}\text { Mean Difference } \\
\text { IV, Random, 95\% CI } \\
-22.00[-27.87,-16.13]\end{array}$} & \multirow{2}{*}{\multicolumn{3}{|c|}{$\begin{array}{l}\text { Mean Difference } \\
\text { IV, Random, 95\% CI }\end{array}$}} \\
\hline & \multirow{2}{*}{$\begin{array}{c}\text { Mean } \\
72\end{array}$} & \multirow{2}{*}{$\frac{S D}{10}$} & \multirow{2}{*}{$\begin{array}{c}\text { Total } \\
30\end{array}$} & \multirow{2}{*}{$\begin{array}{c}\text { Mean } \\
94\end{array}$} & \multirow{2}{*}{$\frac{S D}{13}$} & \multirow{2}{*}{$\begin{array}{c}\text { Total } \\
30\end{array}$} & & & & & \\
\hline & & & & & & & & & $\longrightarrow$ & & \\
\hline He W 2019 & 62.12 & 1.05 & 53 & 70.26 & 1.08 & 53 & 15.4 & $-8.14[-8.55,-7.73]$ & - & & \\
\hline Hu S 2016 & 63.7 & 5.4 & 62 & 69.2 & 6.4 & 63 & 13.6 & $-5.50[-7.57,-3.43]$ & $\rightarrow$ & & \\
\hline Liu L 2019 & 63.6 & 6.4 & 40 & 69.8 & 5.7 & 40 & 12.7 & $-6.20[-8.86,-3.54]$ & $\square-$ & & \\
\hline Lu DS 2019 & 60.02 & 5.25 & 42 & 69.99 & 6.89 & 42 & 12.7 & $-9.97[-12.59,-7.35]$ & 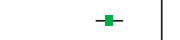 & & \\
\hline Tan X 2019 & 63.5 & 5.7 & 41 & 68.3 & 5.8 & 41 & 13.0 & $-4.80[-7.29,-2.31]$ & $\rightarrow-$ & & \\
\hline Wang QM 2018 & 60.18 & 2.26 & 29 & 73.57 & 5.19 & 29 & 13.7 & $-13.39[-15.45,-11.33]$ & + & & \\
\hline Wang XP 2018 & 64.28 & 6.63 & 30 & 69.25 & 6.47 & 30 & 11.5 & $-4.97[-8.28,-1.66]$ & -- & & \\
\hline Total $(95 \% \mathrm{CI})$ & & & 327 & & & 328 & 100.0 & $-8.71[-10.93,-6.50]$ & 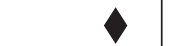 & & \\
\hline $\begin{array}{l}\text { Heterogeneity: } \mathrm{Tau}^{2} \\
\text { Test for overall effec }\end{array}$ & $\begin{array}{l}=8.23 ; C \\
Z=7.72\end{array}$ & $\begin{array}{l}\mathrm{hi}^{2}=6 \\
2(\mathrm{P}<\end{array}$ & $\begin{array}{l}6.92, \mathrm{df} \\
.00001\end{array}$ & $f=7(P$ & $<0.0$ & 001); I & $I^{2}=90 \%$ & -5 & -25 & 25 & 50 \\
\hline
\end{tabular}

FIGURE 5: Forest plot of QQC plus Western medicine treatment versus Western medicine only for HR. QQC: Qili Qiangxin capsule, HR: heart rate.

\begin{tabular}{|c|c|c|c|c|c|c|c|c|c|c|c|}
\hline \multirow{3}{*}{$\begin{array}{l}\text { Study or Subgroup } \\
\text { He W } 2019\end{array}$} & \multicolumn{3}{|c|}{ QQC+Metoprolol } & \multicolumn{3}{|c|}{ Metoprolol } & \multirow{2}{*}{$\begin{array}{l}\text { Weight } \\
(\%)\end{array}$} & \multirow{2}{*}{$\begin{array}{c}\text { Mean Difference } \\
\text { IV, Random, 95\% CI }\end{array}$} & \multirow{2}{*}{\multicolumn{2}{|c|}{$\begin{array}{l}\text { Mean Difference } \\
\text { IV, Random, 95\% CI }\end{array}$}} & \\
\hline & Mean & SD & Total & Mean & $\mathrm{SD}$ & Total & & & & & \\
\hline & 45.02 & 0.16 & 53 & 41.62 & 0.21 & 53 & 30.3 & $3.40[3.33,3.47]$ & & - & \\
\hline Hu S 2016 & 43.8 & 6.5 & 62 & 40.5 & 6.3 & 63 & 11.1 & $3.30[1.06,5.54]$ & & $\multimap$ & \\
\hline Liu Y 2019 & 53.79 & 6.01 & 39 & 47.23 & 5.41 & 39 & 9.4 & $6.56[4.02,9.10]$ & & 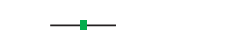 & \\
\hline Lu C 2020 & 44.98 & 4.93 & 53 & 41.87 & 4.56 & 53 & 14.3 & $3.11[1.30,4.92]$ & & $\rightarrow-$ & \\
\hline Lu DS 2019 & 45.68 & 6.52 & 42 & 39.02 & 6.08 & 42 & 8.7 & $6.66[3.96,9.36]$ & & 一 & \\
\hline Tan X 2019 & 58.5 & 7.3 & 41 & 53.6 & 6.7 & 41 & 7.3 & $4.90[1.87,7.93]$ & & - & \\
\hline Wang QM 2018 & 46.18 & 0.58 & 29 & 43.72 & 3.57 & 29 & 19.0 & $2.46[1.14,3.78]$ & & $\rightarrow-$ & \\
\hline Total $(95 \% \mathrm{CI})$ & & & 319 & & & 320 & 100.0 & $3.86[2.92,4.80]$ & & $\vartheta$ & \\
\hline \multicolumn{9}{|c|}{ Heterogeneity: $\mathrm{Tau}^{2}=0.76 ; \mathrm{Chi}^{2}=14.57, \mathrm{df}=6(\mathrm{P}=0.02) ; \mathrm{I}^{2}=59 \%$} & -10 & 10 & 20 \\
\hline \multicolumn{9}{|c|}{ Test for overall effect: $Z=8.06(P<0.00001)$} & Metoprolol & QQC+Metoprolol & \\
\hline
\end{tabular}

(a)

Figure 6: Continued. 


\begin{tabular}{|c|c|c|c|c|c|c|c|c|c|c|}
\hline \multirow{3}{*}{$\begin{array}{l}\text { Study or Subgroup } \\
\text { Feng CY } 2015\end{array}$} & \multicolumn{3}{|c|}{ QQC+Carvedilol } & \multicolumn{3}{|c|}{ Carvedilol } & \multirow{3}{*}{$\begin{array}{c}\text { Weight } \\
(\%)\end{array}$} & \multirow{3}{*}{$\begin{array}{c}\text { Mean Difference } \\
\text { IV, Random, 95\% CI } \\
8.40[5.16,11.64]\end{array}$} & \multirow{2}{*}{\multicolumn{2}{|c|}{$\begin{array}{c}\text { Mean Difference } \\
\text { IV, Random, 95\% CI }\end{array}$}} \\
\hline & \multirow{2}{*}{$\begin{array}{c}\text { Mean } \\
52.2\end{array}$} & \multirow{2}{*}{$\begin{array}{l}\text { SD } \\
7.2\end{array}$} & \multirow{2}{*}{$\begin{array}{c}\text { Total } \\
30\end{array}$} & \multirow{2}{*}{$\begin{array}{c}\text { Mean } \\
43.8\end{array}$} & \multirow{2}{*}{$\begin{array}{l}\mathrm{SD} \\
5.5\end{array}$} & \multirow{2}{*}{$\frac{\text { Total }}{30}$} & & & & \\
\hline & & & & & & & & & & $\rightarrow-$ \\
\hline Meng JC 2019 & 53.16 & 7.91 & 53 & 45.43 & 7.65 & 53 & 19.8 & $7.73[4.77,10.69]$ & & - \\
\hline Ren WW 2018 & 49.82 & 4.58 & 39 & 41.35 & 3.68 & 39 & 21.0 & $8.47[6.63,10.31]$ & & - \\
\hline Yang QY 2019 & 49.67 & 5.13 & 39 & 41.24 & 3.97 & 39 & 20.8 & $8.43[6.39,10.47]$ & & $-1+$ \\
\hline Zhou YF 2018 & 56.99 & 10.01 & 43 & 34.22 & 6.47 & 42 & 19.0 & $22.77[19.20,26.34]$ & & $\rightarrow-$ \\
\hline Total $(95 \% \mathrm{CI})$ & & & 204 & & & 203 & 100.0 & $11.02[6.68,15.36]$ & & \\
\hline \multicolumn{2}{|c|}{ Test for overall effect: $\mathrm{Z}=4.98(\mathrm{P}<0.00001)$} & & & & & & & & \multicolumn{2}{|c|}{ 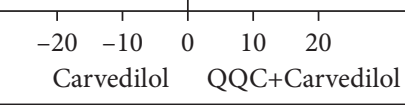 } \\
\hline \multicolumn{11}{|c|}{ (b) } \\
\hline Study or Subgroup & $\begin{array}{l}\text { QQC+ } \\
\text { Mean }\end{array}$ & $\begin{array}{c}\text { Trimet: } \\
\text { SD }\end{array}$ & $\begin{array}{c}\text { azidine } \\
\text { Total }\end{array}$ & $\begin{array}{l}\text { Trim } \\
\text { Mean }\end{array}$ & $\begin{array}{c}\text { netazi } \\
\mathrm{SD}\end{array}$ & $\begin{array}{l}\text { dine } \\
\text { Total }\end{array}$ & $\begin{array}{c}\text { Weight } \\
(\%)\end{array}$ & $\begin{array}{c}\text { Mean Difference } \\
\text { IV, Random, 95\% CI }\end{array}$ & \multicolumn{2}{|c|}{$\begin{array}{c}\text { Mean Difference } \\
\text { IV, Random, 95\% CI }\end{array}$} \\
\hline Chen WQ 2012 & 45.1 & 6.8 & 60 & 42.2 & 7.3 & 60 & 12.8 & $2.90[0.38,5.42]$ & & - \\
\hline Huang XY 2013 & 57.89 & 3.21 & 30 & 51.19 & 3.23 & 30 & 13.5 & $6.70[5.07,8.33]$ & & - \\
\hline Li WH 2015 & 45.2 & 6.7 & 41 & 42.3 & 7.5 & 41 & 12.3 & $2.90[-0.18,5.98]$ & & 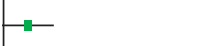 \\
\hline Liu LP 2018 & 53.9 & 6.1 & 50 & 40.6 & 5 & 50 & 13.1 & $13.30[11.11,15.49]$ & & - \\
\hline Wang XF 2018 & 56.97 & 7.26 & 46 & 41.56 & 6.52 & 46 & 12.5 & $15.41[12.59,18.23]$ & & $\rightarrow-$ \\
\hline Yin YS 2015 & 46 & 5 & 42 & 39 & 5 & 42 & 13.1 & $7.00[4.86,9.14]$ & & $-\square-$ \\
\hline Zhang HY 2015 & 47.75 & 6.88 & 49 & 42.69 & 5.86 & 49 & 12.8 & $5.06[2.53,7.59]$ & & $\rightarrow-$ \\
\hline Zhou FZ 2013 & 46.99 & 8 & 19 & 34.7 & 9 & 20 & 9.9 & $12.29[6.95,17.63]$ & & - \\
\hline Total $(95 \% \mathrm{CI})$ & & & 337 & & & 338 & 100.0 & $8.08[4.99,11.17]$ & & \\
\hline \multicolumn{9}{|c|}{$\begin{array}{l}\text { Heterogeneity: } \mathrm{Tau}^{2}=17.74 ; \mathrm{Chi}^{2}=86.00, \mathrm{df}=7(\mathrm{P}<0.00001) ; \mathrm{I}^{2}=92 \% \\
\text { Test for overall effect: } \mathrm{Z}=5.13(\mathrm{P}<0.00001)\end{array}$} & $\begin{array}{lll}-20 & -10 & 0 \\
\text { Trimetazidine }\end{array}$ & $\begin{array}{ccc}0 & 10 & 20 \\
\text { QQC+Trimetaz }\end{array}$ \\
\hline
\end{tabular}

(c)

FIGURE 6: Forest plot of QQC plus Western medicine treatment versus Western medicine only for LVEF (part 1). QQC: Qili Qiangxin capsule, LVEF: left ventricular ejection fractions.

\begin{tabular}{|c|c|c|c|c|c|c|c|c|c|c|c|c|}
\hline \multirow{3}{*}{$\begin{array}{l}\text { Study or Subgroup } \\
\text { Li CY } 2020\end{array}$} & \multicolumn{3}{|c|}{ QQC+SVS } & \multicolumn{3}{|c|}{ SVS } & \multirow{2}{*}{$\begin{array}{l}\text { Weight } \\
(\%)\end{array}$} & \multirow{2}{*}{$\begin{array}{l}\text { Mean Difference } \\
\text { IV, Random, 95\% CI }\end{array}$} & \multirow{2}{*}{\multicolumn{4}{|c|}{$\begin{array}{c}\text { Mean Difference } \\
\text { IV, Random, 95\% CI }\end{array}$}} \\
\hline & Mean & $\mathrm{SD}$ & Total & Mean & SD & Total & & & & & & \\
\hline & 52.17 & 9.34 & 35 & 41.01 & 8.55 & 35 & 15.4 & $11.16[6.96,15.36]$ & & & $\longrightarrow-$ & \\
\hline Su CB 2020 & 53.08 & 3.82 & 37 & 49.26 & 4.03 & 37 & 27.3 & $3.82[2.03,5.61]$ & & & -1 & \\
\hline Wang L 2019 & 58.04 & 5.96 & 57 & 48.89 & 6.82 & 57 & 24.3 & $9.15[6.80,11.50]$ & & & $-1-$ & \\
\hline Xu JF 2020 & 49.68 & 0.37 & 50 & 44.23 & 0.36 & 50 & 33.0 & $5.45[5.31,5.59]$ & & & $\dot{0}$ & \\
\hline \multicolumn{3}{|l|}{ Total $(95 \% \mathrm{CI})$} & 179 & & & 179 & 100.0 & $6.78[4.53,9.04]$ & & & & \\
\hline \multicolumn{9}{|c|}{ Heterogeneity: $\mathrm{Tau}^{2}=4.01 ; \mathrm{Chi}^{2}=19.80, \mathrm{df}=3(\mathrm{P}=0.0002) ; \mathrm{I}^{2}=85 \%$} & -20 & -10 & 10 & 20 \\
\hline \multicolumn{9}{|c|}{ Test for overall effect: $\mathrm{Z}=5.90(\mathrm{P}<0.00001)$} & & SVS & QQC+SVS & \\
\hline
\end{tabular}

(a)

Figure 7: Continued. 


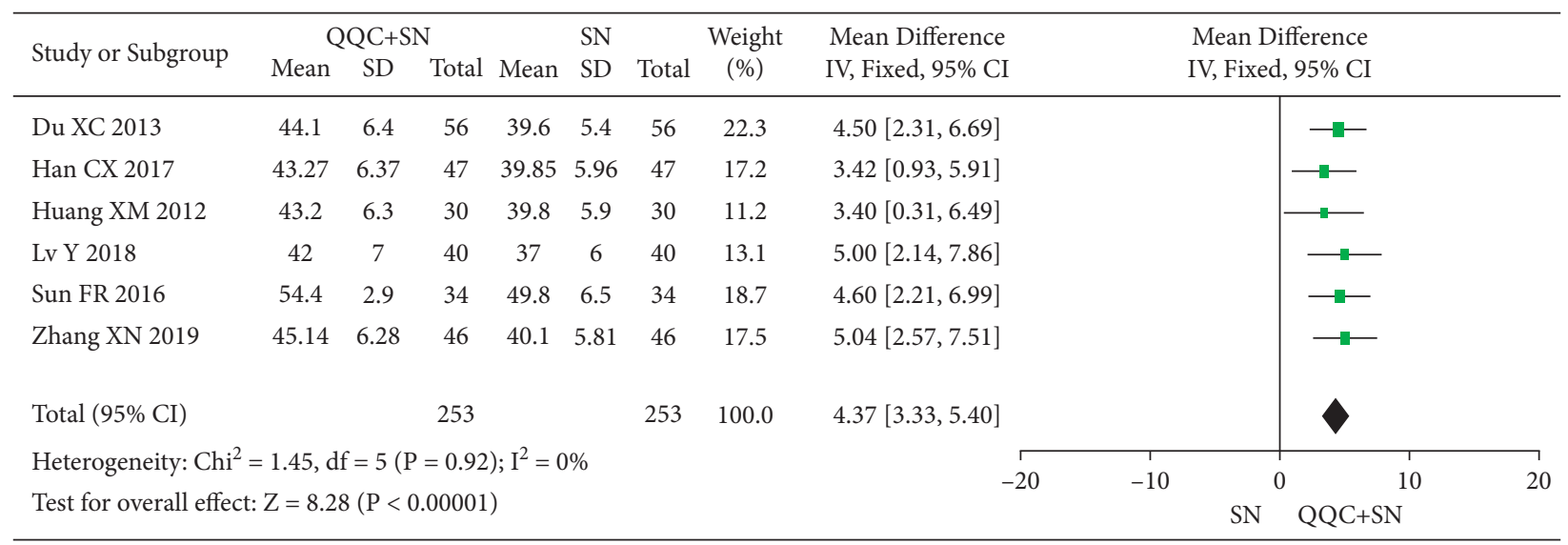

(b)

FIGURE 7: Forest plot of QQC plus Western medicine treatment versus Western medicine only for LVEF (part 2). QQC: Qili Qiangxin capsule, SVS: sacubitril valsartan sodium, SN: sodium nitroprusside, LVEF: left ventricular ejection fractions.

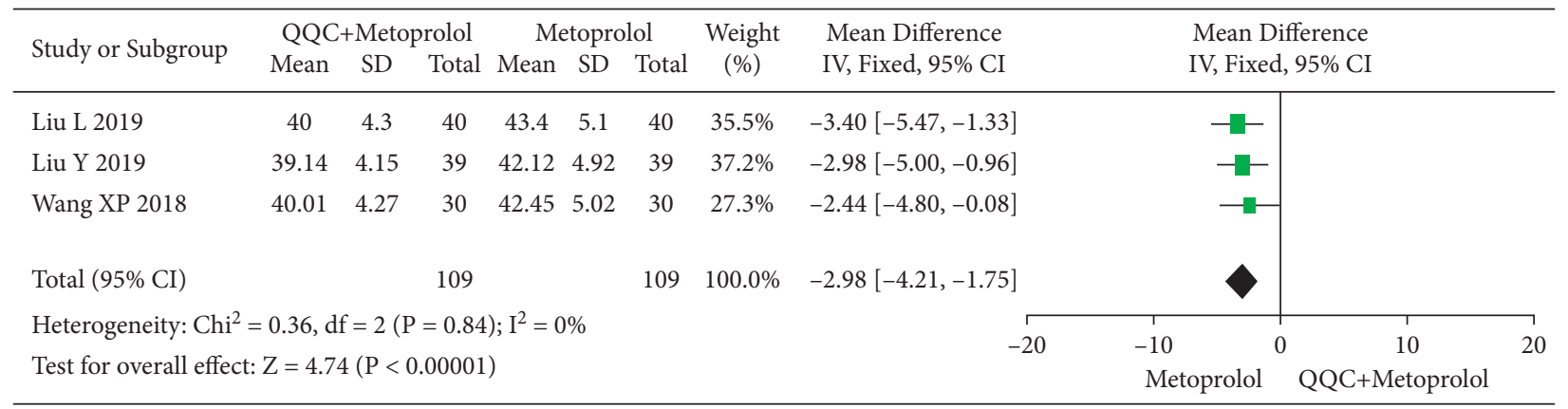

(a)

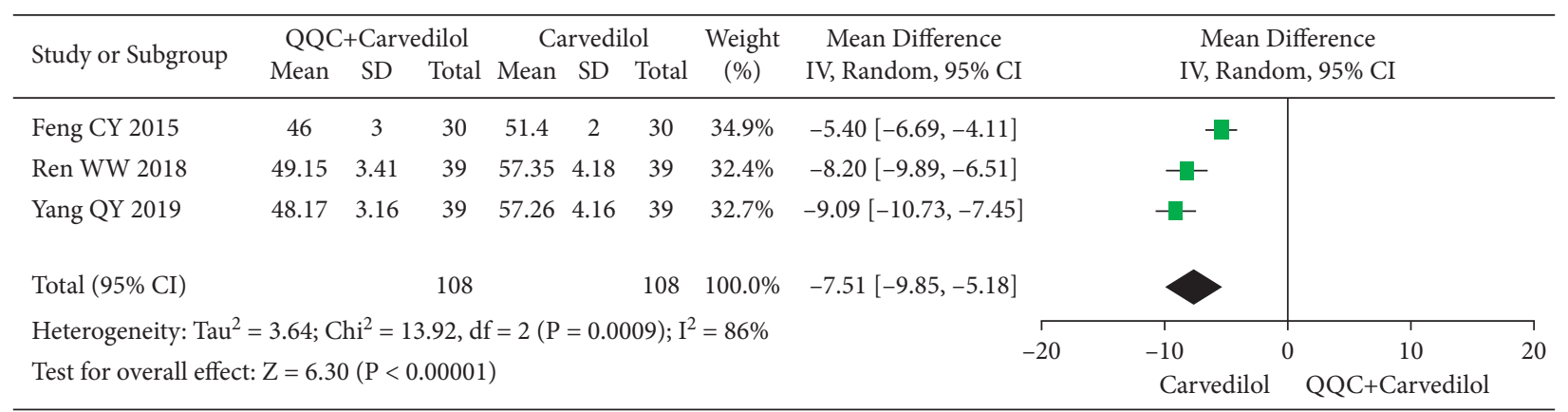

(b)

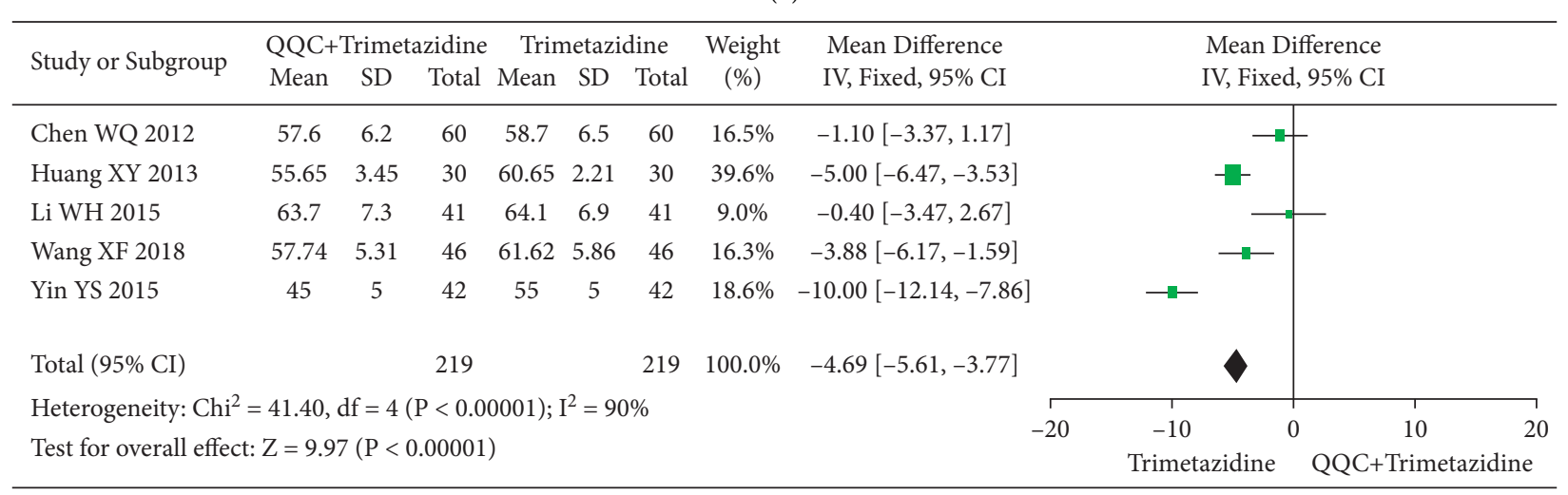

(c)

Figure 8: Continued. 


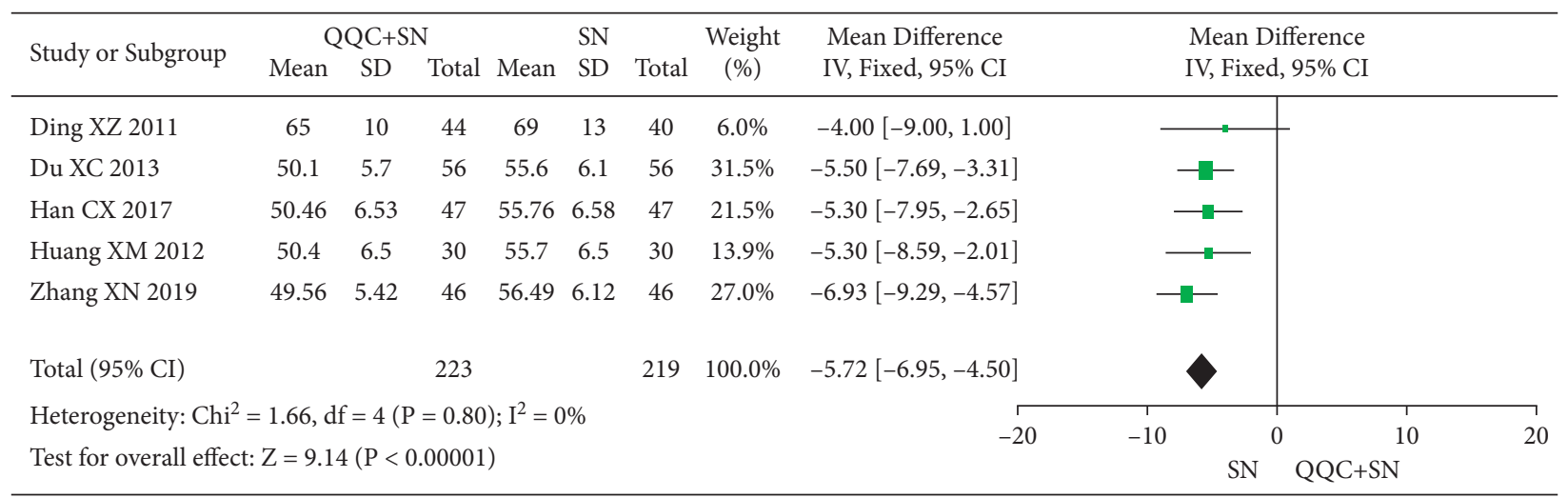

(d)

FIGURE 8: Forest plot of QQC plus Western medicine treatment versus Western medicine only for LVEDD. QQC: Qili Qiangxin capsule, SN: sodium nitroprusside, LVEDD: left ventricular end-diastolic dimension.

\begin{tabular}{|c|c|c|c|c|c|c|c|c|c|c|}
\hline \multirow{3}{*}{$\begin{array}{l}\text { Study or Subgroup } \\
\text { Liu LP } 2018\end{array}$} & \multicolumn{3}{|c|}{ QQC+Trimetazidine } & \multicolumn{3}{|c|}{ Trimetazidine } & \multirow{3}{*}{$\begin{array}{c}\text { Weight } \\
(\%)\end{array}$} & \multirow{3}{*}{$\begin{array}{c}\text { Mean Difference } \\
\text { IV, Random, 95\% CI } \\
-34.70[-39.75,-29.65]\end{array}$} & \multirow{2}{*}{\multicolumn{2}{|c|}{$\begin{array}{l}\text { Mean Difference } \\
\text { IV, Random, 95\% CI }\end{array}$}} \\
\hline & \multirow{2}{*}{$\begin{array}{c}\text { Mean } \\
88.9\end{array}$} & \multirow{2}{*}{$\begin{array}{l}\text { SD } \\
10.2\end{array}$} & \multirow{2}{*}{$\begin{array}{c}\text { Total } \\
50\end{array}$} & \multirow{2}{*}{$\begin{array}{c}\text { Mean } \\
123.6\end{array}$} & \multirow{2}{*}{$\frac{S D}{15.1}$} & \multirow{2}{*}{$\begin{array}{c}\text { Total } \\
50\end{array}$} & & & & \\
\hline & & & & & & & & & - & \\
\hline Wang XF 2018 & 112.48 & 11.35 & 46 & 131.59 & 19.4 & 46 & 37.4 & $-19.11[-25.61,-12.61]$ & - & \\
\hline Zhou FZ 2013 & 129.1 & 25 & 19 & 148.2 & 29 & 20 & 23.6 & $-19.10[-36.07,-2.13]$ & $\longrightarrow$ & \\
\hline Total $(95 \% \mathrm{CI})$ & & & 115 & & & 116 & 100.0 & $-25.19[-37.69,-12.68]$ & & \\
\hline Heterogeneity: Tau & $=97.77 ;$ & $\mathrm{Chi}^{2}=1$ & $14.99, \mathrm{c}$ & $\mathrm{df}=2(1$ & $P=0$. & $006) ; \mathrm{I}$ & $I^{2}=87 \%$ & -50 & -25 & 25 \\
\hline Test for overall effec & $\mathrm{Z}=3.95$ & $5(\mathrm{P}<0$ & $.0001)$ & & & & & & Trimetazidine & QQC+Trimetazidine \\
\hline
\end{tabular}

FIgURE 9: Forest plot of QQC plus Western medicine treatment versus Western medicine only for LVEDV. QQC: Qili Qiangxin capsule, LVEDV: left ventricular end-diastolic volume.

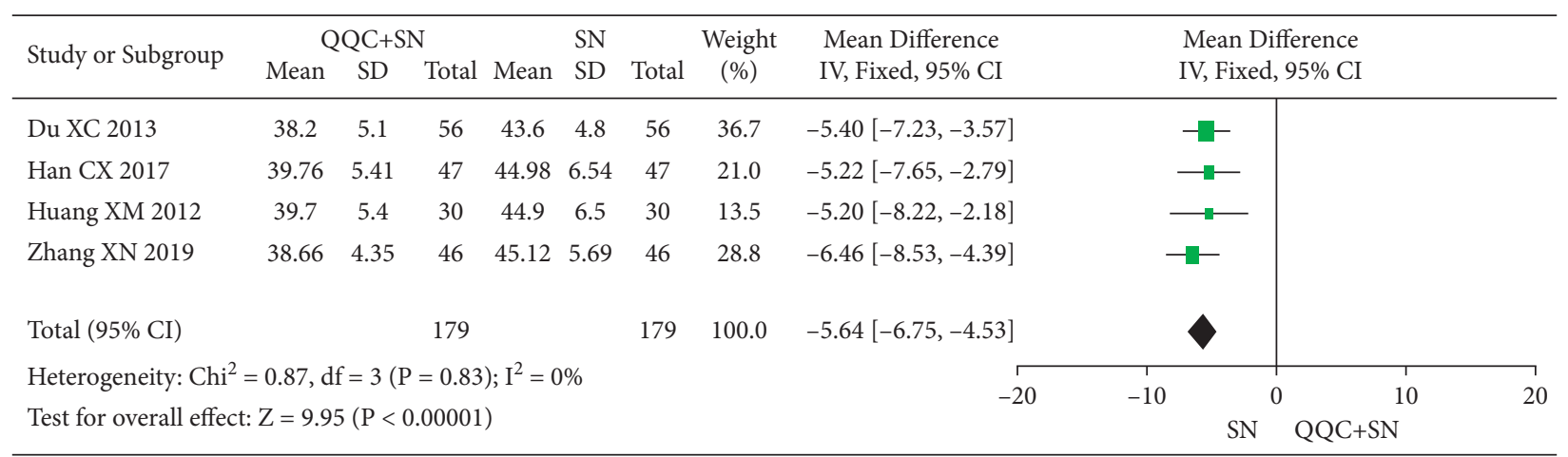

FIGURE 10: Forest plot of QQC plus Western medicine treatment versus Western medicine only for LVESD. QQC: Qili Qiangxin capsule, $\mathrm{SN}$ : sodium nitroprusside, LVESD: left ventricular end-systolic diameter.

of TCM with western medicine has been developed as a novel therapeutic approach for the treatment of $\mathrm{CHF}$. It has the unique advantage of reducing the adverse reactions compared with Western medicine alone [21]. In this study, we revealed that compared with Western medicine alone, the combination of QQC with Western medicine for $\mathrm{CHF}$ showed better clinical efficiency. However, more evidence was required to validate the advantage of QQC plus Western medicine on reducing the occurrence of adverse effects.
QQC is a traditional Chinese medical formulation, which has been demonstrated to improve cardiac function and urine volume of CHF patients. Previous studies have found that the possible mechanisms of QQC in the treatment of $\mathrm{CHF}$ might be connected with reversing the increases of both AQP2 and pS256-AQP2 expression and involving the inhibition of $\mathrm{V} 2 \mathrm{R}$ and $\mathrm{AT} 1 \mathrm{R}[13,73]$. The research of the effects of QQC on cardiac function in rats with myocardial infarction suggested that it improved 


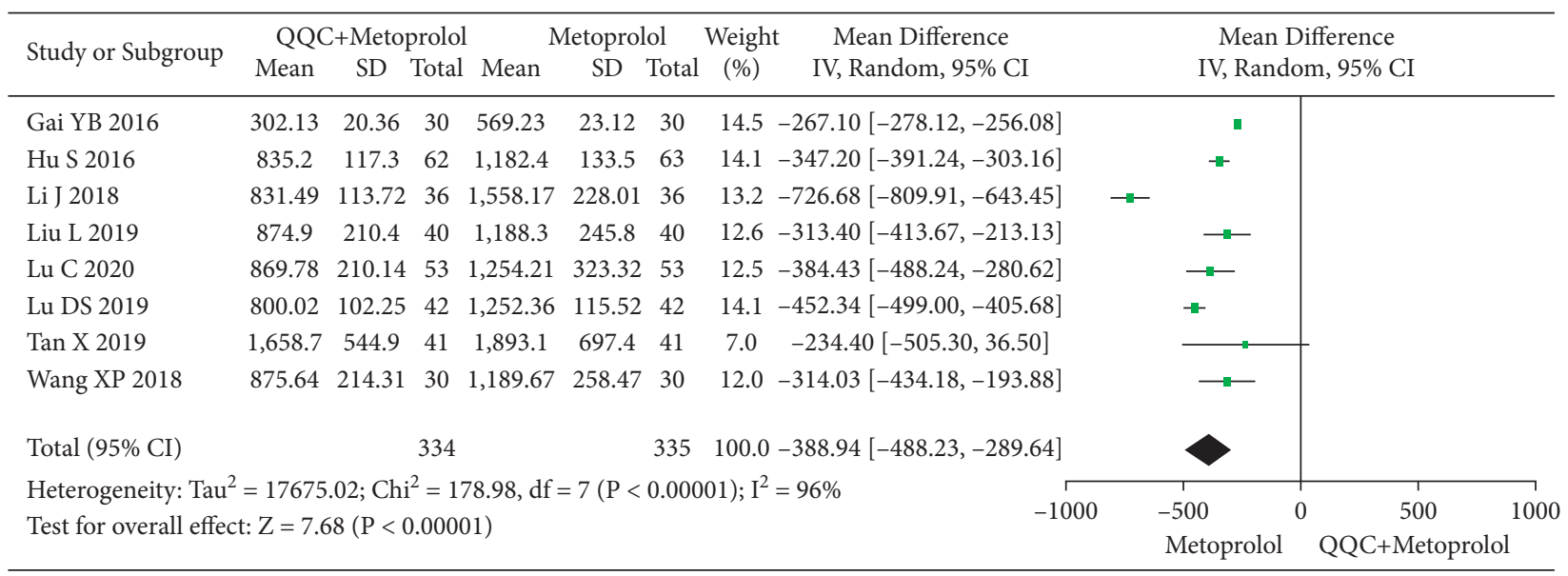

(a)

\begin{tabular}{|c|c|c|c|c|c|c|c|c|c|c|c|}
\hline \multirow{3}{*}{$\begin{array}{l}\text { Study or Subgroup } \\
\text { Liu YY } 2018\end{array}$} & \multicolumn{3}{|c|}{ QQC+Bisoprolol } & \multicolumn{3}{|c|}{ Bisoprolol } & \multirow{2}{*}{$\begin{array}{l}\text { Weight } \\
(\%)\end{array}$} & \multirow{2}{*}{$\begin{array}{c}\text { Mean Difference } \\
\text { IV, Random, 95\% CI }\end{array}$} & \multirow{2}{*}{\multicolumn{3}{|c|}{$\begin{array}{l}\text { Mean Difference } \\
\text { IV, Random, 95\% CI }\end{array}$}} \\
\hline & Mean & $\mathrm{SD} 7$ & Total & Mean & $\mathrm{SD}$ & Total & & & & & \\
\hline & $1,163.06$ & 213.62 & 49 & $1,705.39$ & 293.61 & 49 & 31.6 & $-542.33[-644.00,-440.66]$ & $\rightarrow-$ & & \\
\hline Zhang M 2019 & 542.87 & 99.63 & 39 & 648.35 & 102.65 & 39 & 34.2 & $-105.48[-150.38,-60.58]$ & - & & \\
\hline Zhang XW 2016 & 543.21 & 98.46 & 40 & 645.47 & 103.24 & 40 & 34.2 & $-102.26[-146.47,-58.05]$ & $-\frac{\mathrm{n}}{\mathrm{B}}$ & & \\
\hline Total $(95 \% \mathrm{CI})$ & & & 128 & & & 128 & 100.0 & $-242.41[-428.59,-56.24]$ & & & \\
\hline $\begin{array}{l}\text { Heterogeneity: } \mathrm{Tau}^{2} \\
\text { Test for overall effec }\end{array}$ & $\begin{array}{l}2=25864 . \\
\text { ct: } Z=2.5\end{array}$ & $\begin{array}{l}83 ; \mathrm{Chi}^{2} \\
5(\mathrm{P}=0\end{array}$ & $\begin{array}{l}\mathrm{i}^{2}=6 \\
0.01)\end{array}$ & $5.21, \mathrm{df}=$ & $=2(\mathrm{P}<0$. & 0.0000 & 1); $\mathrm{I}^{2}=$ & -1000 & $\begin{array}{l}-500 \quad 0 \\
\text { Bisoprolol }\end{array}$ & $\begin{array}{cc}500 \\
\text { QCC+Bisoprolol }\end{array}$ & 1000 \\
\hline
\end{tabular}

(b)

FIGURE 11: Forest plot of QQC plus Western medicine treatment versus Western medicine only for BNP. QQC: Qili Qiangxin capsule, BNP: brain natriuretic peptide.

\begin{tabular}{|c|c|c|c|c|c|c|c|c|c|c|c|c|}
\hline \multirow{3}{*}{$\begin{array}{l}\text { Study or Subgroup } \\
\text { Li WH } 2015\end{array}$} & \multicolumn{3}{|c|}{ QQC+Trimetazidine } & \multicolumn{3}{|c|}{ Trimetazidine } & \multirow{3}{*}{$\begin{array}{c}\text { Weight } \\
(\%)\end{array}$} & \multirow{3}{*}{$\begin{array}{c}\begin{array}{c}\text { Mean Difference } \\
\text { IV, Random, 95\% CI }\end{array} \\
-0.14[-0.22,-0.06]\end{array}$} & \multirow{2}{*}{\multicolumn{4}{|c|}{$\begin{array}{c}\text { Mean Difference } \\
\text { IV, Random, 95\% CI }\end{array}$}} \\
\hline & \multirow{2}{*}{$\begin{array}{c}\text { Mean } \\
0.52\end{array}$} & \multirow{2}{*}{$\frac{S D}{0.17}$} & \multirow{2}{*}{$\begin{array}{c}\text { Total } \\
41\end{array}$} & \multirow{2}{*}{$\begin{array}{c}\text { Mean } \\
0.66\end{array}$} & \multirow{2}{*}{$\frac{S D}{0.18}$} & \multirow{2}{*}{$\begin{array}{c}\text { Total } \\
41\end{array}$} & & & & & & \\
\hline & & & & & & & & & & $\square$ & & \\
\hline Wei HC 2015 & 1.33 & 0.24 & 42 & 2.36 & 0.41 & 42 & 33.2 & $-1.03[-1.17,-0.89]$ & & - 는 & & \\
\hline Zhang HY 2015 & 1.76 & 0.38 & 49 & 2.01 & 0.42 & 49 & 33.0 & $-0.25[-0.41,-0.09]$ & & - - & & \\
\hline \multicolumn{3}{|l|}{ Total $(95 \% \mathrm{CI})$} & 132 & & & 132 & 100.0 & $-0.47[-1.01,0.07]$ & & & & \\
\hline \multicolumn{9}{|c|}{ Heterogeneity: $\mathrm{Tau}^{2}=0.23 ; \mathrm{Chi}^{2}=116.22, \mathrm{df}=2(\mathrm{P}<0.00001) ; \mathrm{I}^{2}=98 \%$} & -2 & -1 & 1 & 2 \\
\hline \multicolumn{9}{|c|}{ Test for overall effect: $\mathrm{Z}=1.70(\mathrm{P}=0.09)$} & & letazidine & QCC+Tri & netazidine \\
\hline
\end{tabular}

(a)

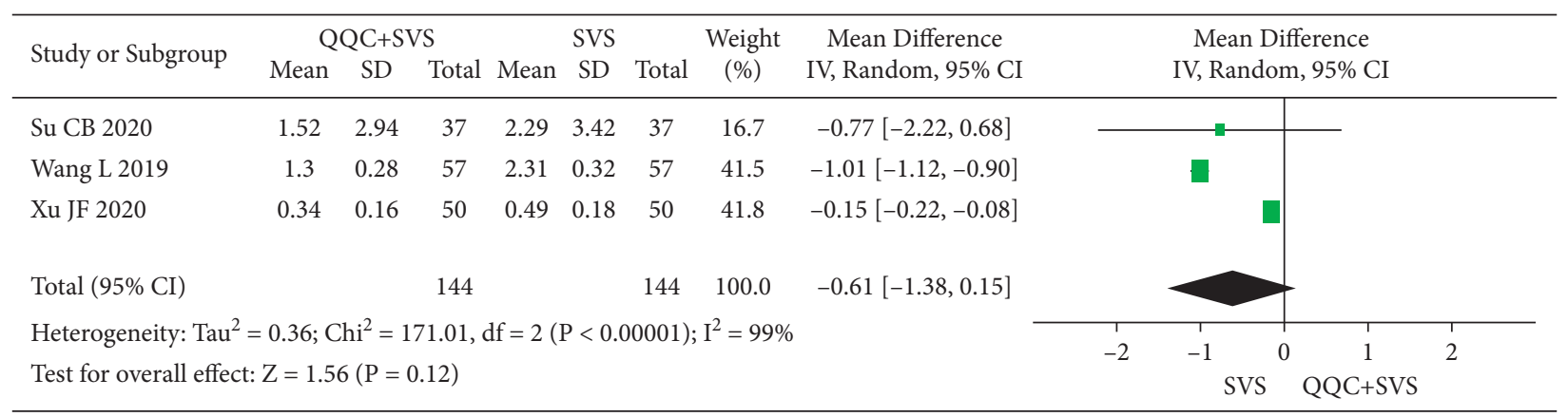

(b)

FIgURE 12: Forest plot of QQC plus Western medicine treatment versus Western medicine only for NT-proBNP. QQC: Qili Qiangxin capsule, SVS: sacubitril valsartan sodium, NT-proBNP: N-terminal pro-B-type natriuretic peptide. 


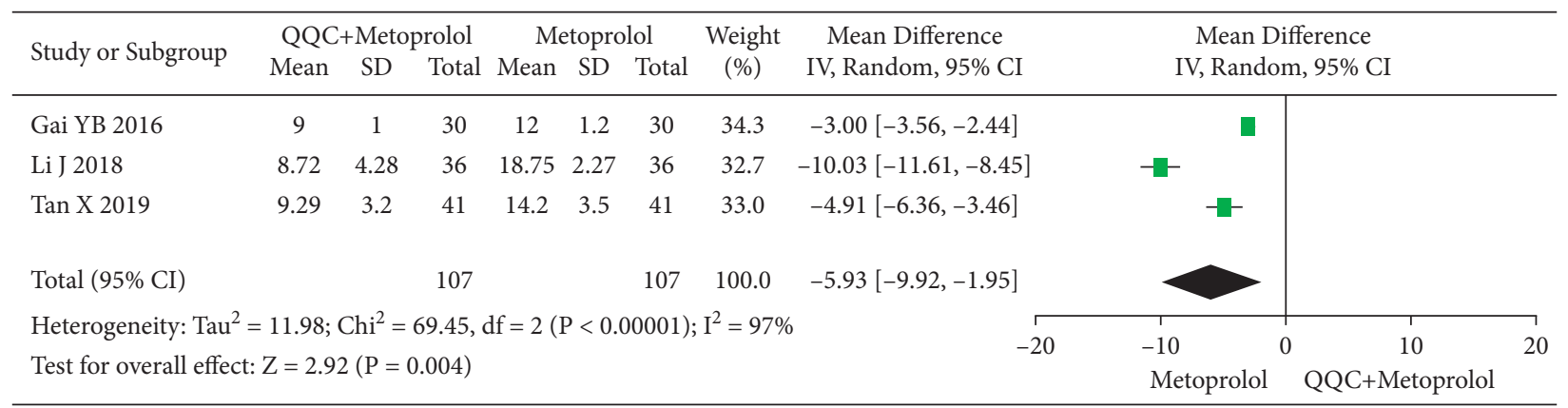

FIgURE 13: Forest plot of QQC plus Western medicine treatment versus Western medicine only for Hs-cTnT. QQC: Qili Qiangxin capsule, Hs-cTnT: high-sensitivity cardiac troponin T.

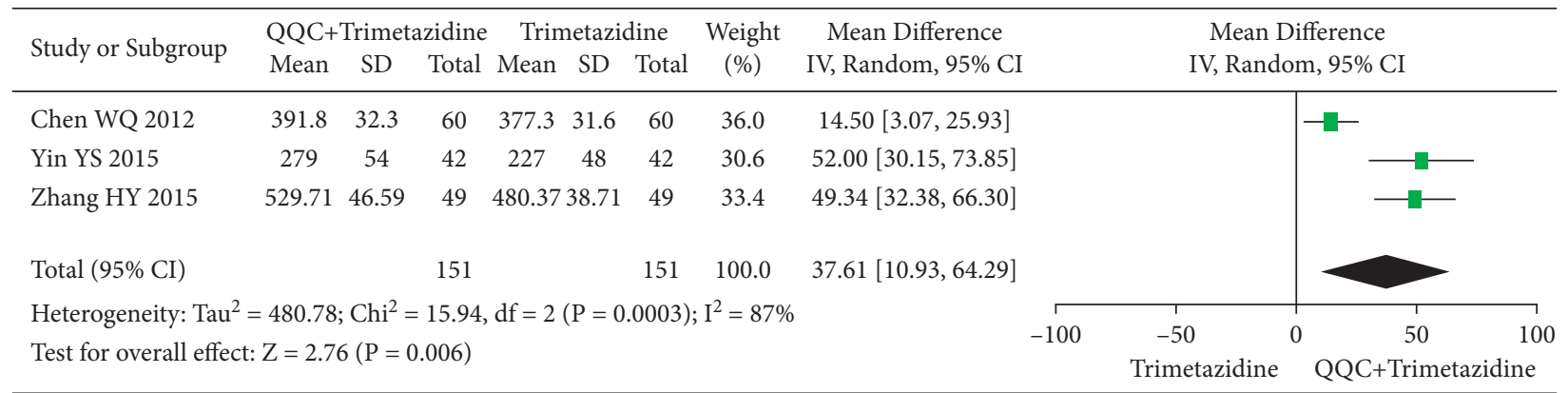

FIGURE 14: Forest plot of QQC plus Western medicine treatment versus Western medicine only for 6MWT. QQC: Qili Qiangxin capsule, 6MWT: 6-min walk test.

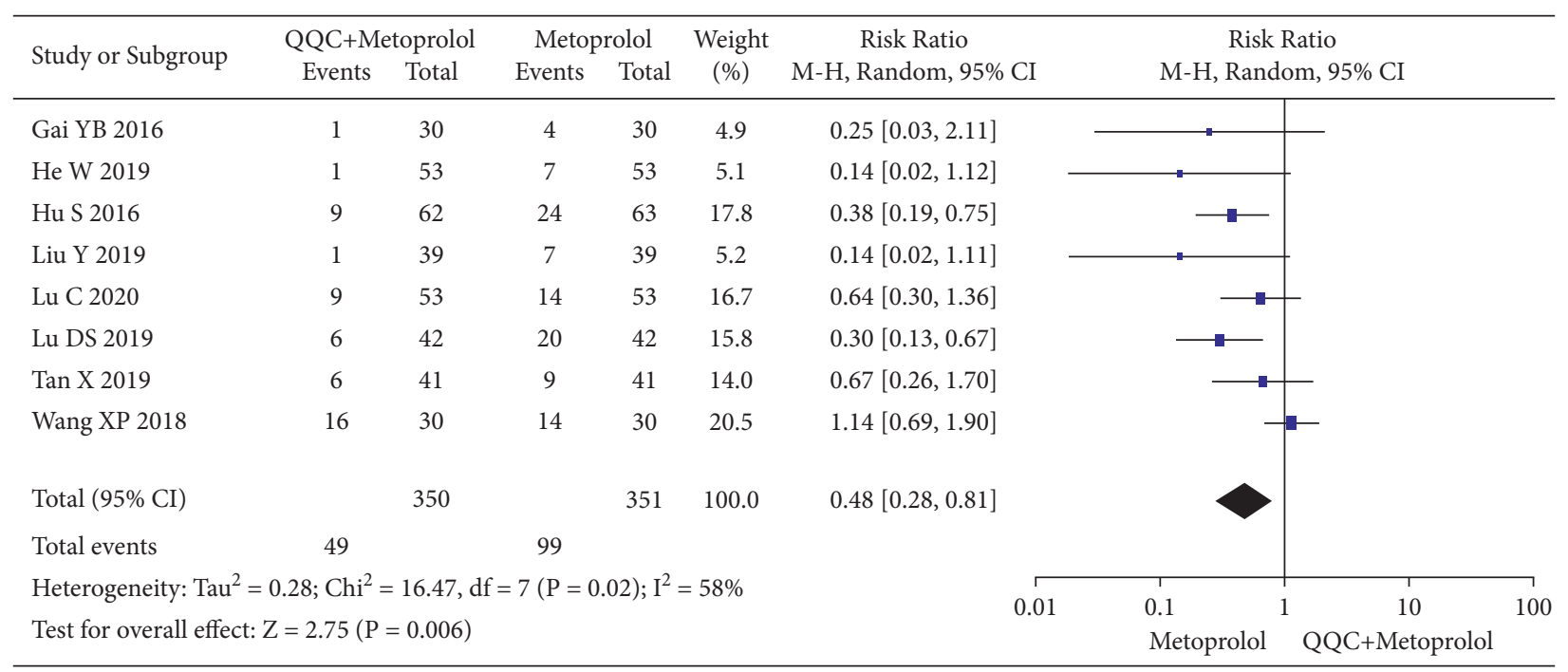

(a)

Figure 15: Continued. 


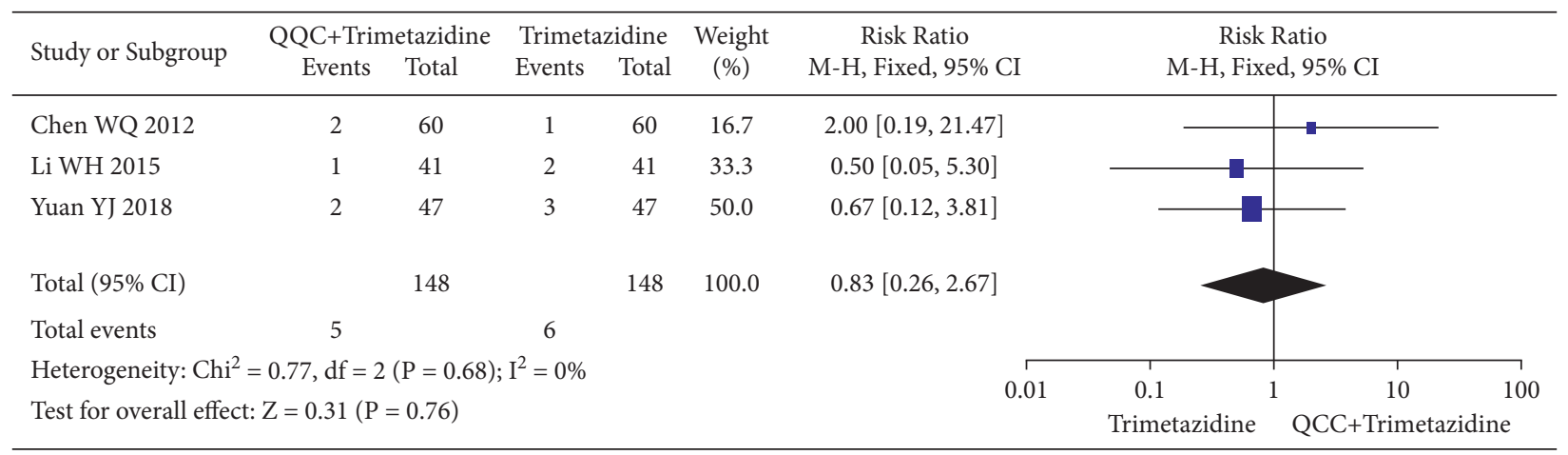

(b)

FIgURE 15: Forest plot of QQC plus Western medicine treatment versus Western medicine only for adverse effects. QQC: Qili Qiangxin capsule.

cardiac function by keeping the balance between proinflammatory and anti-inflammatory of cardiomyocytes [74]. The LVEF, LVEDD, LVEDV, LVESD, BNP, NT-proBNP, Hs-cTnT, and 6MWT are important indicators to reflect the cardiac function of CHF patients $[17,72,75]$. The results of this research showed that compared with Western medicine alone, the combination of QQC with Western medicine exerted positive effects on improving efficacy of $\mathrm{CHF}$, with increased level of LVEF, LVEDD, and 6MWT and reduced level of HR, LVESD, BNP, and Hs-cTnT of patients with $\mathrm{CHF}$. This research also suggested that QQC could improve the quality of life in patients with $\mathrm{CHF}$, which was in agreement with the research in 2016 [21].

In our research, there is high heterogeneity in the metaanalysis of LVEDV, BNP, NT-proBNP, Hs-cTnT, 6MWT, and adverse effects. The level of cardiac function index is influenced by the age and gander. Moreover, etiology (type 2 diabetes mellitus, obesity, valvular heart disease, and others) and underlying disease (depression, kidney disease, sleep disordered, and others) also significantly affected the cardiovascular system [76]. These factors have not been strictly described and controlled in the included studies, which may lead to the high heterogeneity in the meta-analysis of LVEDV, BNP, NT-proBNP, Hs-cTnT, 6MWT, and adverse effects.

This research comprehensively identified the relevant literature, developed evaluation plans, and strictly implemented those plans. These methodological advantages can improve the accuracy and clinical applicability of the results of this study. However, there are several limitations in this research: (1) few studies included for meta-analysis of each indicator may lead to publication bias; (2) the description of allocation concealment is inadequate; (3) the blinding of participants personnel and outcome assessment of included studies are unclear; (4) the overall risk of bias in the included studies was generally high; (5) all subjects included in this study belong to China, it cannot account for ethnic and regional differences. Therefore, more high quality studies were required to find more convincing proof. We recommend researchers report in full their trial methodology such as random sequence generation, allocation concealment, and internationally recognized diagnosis of $\mathrm{CHF}$ in future publications.

\section{Conclusion}

This meta-analysis demonstrated that, compared with Western medicine alone, the combination of QQC and Western medicine exerted more positive effects on improving efficacy and increasing the level of LVEF, LVEDD, and 6MWT, as well as reducs the adverse effects and the level of HR, LVESD, BNP, Hs-cTnT of patients with CHF. The results further proved the efficacy and safety of QQC for $\mathrm{CHF}$ patients. However, there are several limitations in our included studies, and more high quality studies are required to provide more convincing proof.

\section{Data Availability}

All datasets used and analyzed during this study can be available from the corresponding author upon reasonable request.

\section{Conflicts of Interest}

The authors declare that they have no conflicts of interest.

\section{Acknowledgments}

This work was funded by the Natural Science Foundation of Zhejiang Province (no. LY21H290007) and the Scientific Research Foundation of Zhejiang University of Traditional Chinese Medicine (no. 2020ZG45).

\section{Supplementary Materials}

The PRISMA 2020 checklist for the meta-analysis. (Supplementary Materials)

\section{References}

[1] Y. Guo, G. Y. Lip, and A. Banerjee, "Heart failure in east asia," Current Cardiology Reviews, vol. 9, no. 2, pp. 112-122, 2013.

[2] H. Wang, J. Zhang, C.-F. Shi et al., "Combination and distribution characteristics of syndromes related to traditional Chinese medicine in patients with chronic heart failure," Medicine, vol. 99, no. 36, Article ID e21852, 2020. 
[3] S. Sairras, S.-S. Baldew, K. van der Hilst et al., "Heart failure hospitalizations and risk factors among the multi-ethnic population from a middle income country: the Suriname heart failure studies," Journal of the National Medical Association, vol. 113, no. 2, pp. 177-186, 2021.

[4] P. Ponikowski, A. A. Voors, S. D. Anker et al., "ESC guidelines for the diagnosis and treatment of acute and chronic heart failure: the task force for the diagnosis and treatment of acute and chronic heart failure of the european society of cardiology (ESC). Developed with the special contribution of the heart failure association (HFA) of the ESC," European Journal of Heart Failure, vol. 18, no. 8, pp. 891-975, 2016.

[5] A. Head, M. J. Kendall, R. Ferner, and C. Eagles, "Acute effects of beta blockade and exercise on mood and anxiety," British Journal of Sports Medicine, vol. 30, no. 3, pp. 238-242, 1996.

[6] A. Nahid, H. Fataneh, E. Azinmehr, and S. Asieh, "Protective effects of myrtus communis linn fruit and leaf extracts on isoproterenol-induced heart failure in rat," Traditional and Integrative Medicine, vol. 6, no. 2, 2021.

[7] R. Akrami, M. H. Hashempur, A. Tavakoli et al., "Effects of fumaria parviflora $\mathrm{L}$ on uremic pruritus in hemodialysis patients: a randomized, double-blind, placebo-controlled trial," Jundishapur Journal of Natural Pharmaceutical Products, 2016, In press.

[8] M. Seyed Hashemi, M. H. Hashempur, M. H. Lotfi et al., "The efficacy of asafoetida (Ferula assa-foetida oleo-gum resin) versus chlorhexidine gluconate mouthwash on dental plaque and gingivitis: a randomized double-blind controlled trial," European Journal of Integrative Medicine, vol. 29, Article ID 100929, 2019.

[9] B. Brunelli and K. C. Gorson, "The use of complementary and alternative medicines by patients with peripheral neuropathy," Journal of the Neurological Sciences, vol. 218, no. 1-2, pp. 59-66, 2004.

[10] K.-H. Wang, J.-R. Wu, D. Zhang, X.-J. Duan, and M.-W. Ni, "Comparative efficacy of Chinese herbal injections for treating chronic heart failure: a network meta-analysis," BMC Complementary and Alternative Medicine, vol. 18, no. 1, p. 41, 2018.

[11] M. Q. Yang, Y. M. Song, H. Y. Gao, and Y. T. Xue, "Efficacy and safety of fuzi formulae on the treatment of heart failure as complementary therapy: a systematic review and metaanalysis of high-quality randomized controlled trials," Evidence-based Complementary and Alternative Medicine, vol. 2019, Article ID 9728957, 21 pages, 2019.

[12] Y. Wang, X. Zhou, X. Chen et al., "Efficacy and safety of shengmai injection for chronic heart failure: a systematic review of randomized controlled trials," Evidence-based Complementary and Alternative Medicine, vol. 2020, Article ID 9571627, 10 pages, 2020.

[13] X. Cui, J. Zhang, Y. Li et al., "Effects of qili qiangxin capsule on AQP2, V2R, and AT1R in rats with chronic heart failure," Evidence-based Complementary and Alternative Medicine, vol. 2015, Article ID 639450, 11 pages, 2015.

[14] W. Liu, J. Chen, T. Xu et al., "Qiliqiangxin improves cardiac function in spontaneously hypertensive rats through the inhibition of cardiac chymase," American Journal of Hypertension, vol. 25, no. 2, pp. 250-260, 2012.

[15] L. Tao, S. Shen, and X. Li, "Future prospects of Qiliqiangxin on heart failure: epigenetic regulation of regeneration," Frontiers in Genetics, vol. 4, p. 221, 2013.

[16] Y. Zou, L. Lin, Y. Ye et al., "Qiliqiangxin inhibits the development of cardiac hypertrophy, remodeling, and dysfunction during 4 weeks of pressure overload in mice,"
Journal of Cardiovascular Pharmacology, vol. 59, no. 3, pp. 268-280, 2012.

[17] X. Li, J. Zhang, J. Huang et al., "A multicenter, randomized, double-blind, parallel-group, placebo-controlled study of the effects of qili qiangxin capsules in patients with chronic heart failure," Journal of the American College of Cardiology, vol. 62, no. 12, pp. 1065-1072, 2013.

[18] Q. Wang, J. Li, N. Liu, and X. Wang, "Clinical effect observation of qiliqiangxin capsule combined with metoprolol in the treatment of chronic heart failure with heart and kidney yang deficiency," Psychological Documents, vol. 24, no. 13, pp. 128-129, 2018.

[19] X. Tan, X. Gong, and B. Li, "Effect analysis of the treatment of chronic heart failure patients with metopolol combined withLi strong heart capsule," Healthmust-Readmagazine, vol. 12, no. 6, pp. 38-41, 2019.

[20] C. Lu, "Effect of metoprolol combined with qiliqiangxin capsule on Gal-3, HO-1, hcy in patients with chronic heart pailure," Chinese Journal of Rational Drug Use, vol. 17, no. 4, pp. 69-73, 2020.

[21] J. Sun, K. Zhang, W.-J. Xiong et al., "Clinical effects of a standardized Chinese herbal remedy, Qili Qiangxin, as an adjuvant treatment in heart failure: systematic review and meta-analysis," BMC Complementary and Alternative Medicine, vol. 16, no. 1, p. 201, 2016.

[22] M. J. Page, J. E. McKenzie, P. M. Bossuyt et al., "The PRISMA 2020 statement: an updated guideline for reporting systematic reviews," International Journal of Surgery, vol. 88, Article ID 105906, 2021.

[23] H. Liu and H. An, "Clinical observation on treatment of 50 cases of elderly chronic heart failure with qiliqiangxin capsule and digoxin," Chinese Journal of Difficult and Complicated Cases, vol. 7, no. 11, pp. 671-672, 2008.

[24] W. Chen, J. Yu, L. Bai et al., "Clinical study of qiliqiangxin capsule combined with trimetazidine in the treatment of chronic heart failure," Chinese Journal of Diffcult and Complicated Cases, vol. 11, no. 11, pp. 830-832, 2012.

[25] X. Huang, "The influence of sodium nitroprusside joint strong heart capsule for prognosis and quality of life of congestive heart failure patients and its health education countermeasures," Modern Preventive Medicine, vol. 39, no. 17, pp. 4474-4475, 2012.

[26] F. Zhou, "Clinical study of qiliqiangxin capsule combined with trimetazidine in the treatment of congestive heart failure," Chinese Community Doctors, vol. 1, pp. 315-317, 2013.

[27] X. Huang, "Qiliqiangxin capsule combined with trimetazidine in the treatment of 30 cases of chronic heart failure," Zhejiang Journal of Traditional Chinese Medicine, vol. 48, no. 12, p. 922, 2013.

[28] X. Zhen, "Evaluation of the clinical effect of nitroprusside combined with qiliqiangxin capsule in the treatment of chronic congestive heart failure," China Practical Medicine, vol. 8, no. 15, pp. 144-145, 2013.

[29] X. Du, "Evaluation of the effect of sodium nitroprusside combined with qiliqiangxin capsule in the treatment of chronic congestive heart failure," The Journal of Medical Theory and Practice, vol. 26, no. 10, pp. 1304-1305, 2013.

[30] L. Guo, "The therapeutic effect of qiliqiangxin capsule combined with benazepril hydrochloride in the treatment of stable Angina pectoris combined with chronic heart failure," Medical Innovation of China, vol. 11, no. 20, pp. 97-98, 2014.

[31] Z. Feng, "Observation of the curative effect of carvedilol combined with qiliqiangxin capsule in the treatment of 
chronic heart failure," For all Health, vol. 9, no. 18, p. 140, 2015.

[32] H. Wei, "Effects of qiliqiangxin capsules combined with trimetazidine on inflammation and vascular endothelial function in patients with chronic heart failure," Hebei Medical Journal, vol. 37, no. 20, pp. 3067-3069, 2015.

[33] Y. Yin, "Study on the efficacy of qiliqiangxin capsule combined with trimetazidine in the treatment of chronic heart failure," Hebei Medical Journal, vol. 37, no. 18, pp. 2766-2768, 2015.

[34] W. Li, W. Jiang, C. Lv, L. Cao, S. Zhang, and L. Wang, "Clinical observation of qiliqiangxin capsule combined with trimetazidine in the treatment of chronic heart failure," Guide of China Medicine, vol. 13, no. 35, pp. 215-216, 2015.

[35] H. Zhang, "Clinical observation of trimetazidine hydrochloride combined with qiliqiangxin capsule in the treatment of chronic congestive heart failure," Journal of North Pharmacy, vol. 12, no. 5, pp. 33-35, 2015.

[36] X. Zhang, "Observation on the effect of qiliqiangxin capsule combined with bisoprolol in the treatment of chronic heart failure," Henan Medical Research, vol. 25, no. 12, pp. 2247-2248, 2016.

[37] Y. Gai and C. Li, "Qili cardiotonic capsule combined with metoprolol on curative efficacy and influence of plasma BNP and high sensitivity troponin T levels in patients with chronic heart failure," Medical \& Pharmaceutical Journal of Chinese People's Liberation Army, vol. 28, no. 11, pp. 92-94, 2016.

[38] Z. Wang, "Efficacy evaluation of sodium nitroprusside combined with qiliqiangxin capsule in the treatment of chronic congestive heart failure," China Practical Medicine, vol. 11, no. 7, pp. 119-120, 2016.

[39] F. Sun, "Clinical observation on treatment of chronic congestive heart failure with sodium nitroprusside and qiliqiangxin capsule," Clinical Research, vol. 24, no. 8, pp. 82-83, 2016.

[40] S. Hu, "Observation on the curative effect of metoprolol combined with Qiliqiangxin capsule in the treatment of chronic heart failure," Chongqing Yixue, vol. 46, no. 5, pp. 689-691, 2017.

[41] C. Han, "Observation on the effect of sodium nitroprusside combined with qiliqiangxin capsule on chronic congestive heart failure," Henan Medical Research, vol. 26, no. 22, pp. 4071-4072, 2017.

[42] Y. Liu, W. Ren, and N. Wang, "Clinical study of qiliqiangxin capsule combined with bisoprolol in the treatment of 98 cases of chronic heart failure," Special Health, vol. 20, p. 233, 2018.

[43] Y. Zhou, "Analysis of the effect of carvedilol combined with qiliqiangxin capsule on chronic heart failure," Henan Medical Research, vol. 27, no. 18, pp. 3394-3395, 2018.

[44] W. Ren, "Analysis of the efficacy of carvedilol combined with qiliqiangxin capsules in the treatment of chronic heart failure," Inner Mongolia Medical Journal, vol. 50, no. 1, pp. 75-76, 2018.

[45] J. Li, Q. Wang, N. Liu, and X. Wang, "Clinical efficacy and safety of metoprolol combined with Qiliqiangxin capsules in the treatment of chronic heart failure," Diet Health, vol. 5, no. 9, p. 76, 2018.

[46] X. Wang, W. Wu, X. Tian, and J. Zhao, "Clinical evaluation of metoprolol combined with qiliqiangxin capsules in treating chronic heart failure," China Pharmaceuticals, vol. 27, no. 3, pp. 67-69, 2018.

[47] L. Liu, "Influence of trimetazidine dihydrochloride tables combined with qiliqiangxin capsule in the treatment of chronic heart failure," Medical Innovation of China, vol. 36, no. 15, pp. 106-109, 2018.
[48] X. Wang, "Effects of trimetazidine combined with Qiliqiangxin capsules on cardiac function and serum Gal-3 and Copeptin levels in patients with chronic heart failure," Henan Medical Research, vol. 27, no. 13, pp. 2422-2423, 2018.

[49] Y. Yuan, Z. Wang, and K. Feng, "Effect of trimetazidine combined with qiliqiangxin capsule on serum gal-3 and copeptin levels and quality of life in patients with chronic heart failure," Chinese Journal of Rational Drug Use, vol. 15, no. 4, pp. 49-51, 2018.

[50] Y. Wang and Y. Wang, "A randomized double-blind study and electrocardiogram analysis of ivabradine combined with qiliqiangxin capsule in the treatment of 51 patients with chronic heart failure," Cinical Research, vol. 26, no. 10, pp. 136-138, 2018.

[51] C. Wang, "Effect of qili qiangxin capsule combined with levocarnitine on serum IL-6, IL-10 and TNF- $\alpha$ in chronic congestive heart failure," Journal of Liaoning University of TCM, vol. 20, no. 10, pp. 137-140, 2018.

[52] Y. Lv, "Efficacy evaluation of sodium nitroprusside combined with qiliqiangxin capsule in the treatment of chronic congestive heart failure," Clinical Research, vol. 26, no. 1, pp. 171-172, 2018.

[53] M. Zhang, N. Wang, and Y. Li, "Analysis of the effect of qiliqiangxin capsule combined with bisoprolol in the treatment of chronic heart failure," Healthful Friend, vol. 10, pp. 175-176, 2019.

[54] B. Wu, C. Su, Z. Liu et al., "Clinical obseration of qiliqiangxin capsule combined with coenzyme q10 in the treatment of chronic heart failure," Journal of Guangxi Medical University, vol. 37, no. 6, pp. 662-665, 2019.

[55] Q. Yang, "Effect analysis of carvedilol combined with qilu qiangxin capsule on chronic heart failure," Cardiovascular Disease Electronic Journal of Integrated Traditional Chinese and Wetern Medicine, vol. 7, no. 26, pp. 8-9, 2019.

[56] J. Meng, "Efficacy of qiliqiangxin capsule and carvedilol on chronic heart failure of coronary heart disease," Henan Medical Research, vol. 28, no. 10, pp. 1864-1865, 2019.

[57] D. Lu, "Metoprolol combined with Qiliqiangxin capsule in the treatment of 42 cases of chronic heart failure," China's $\mathrm{Na}$ turopathy, vol. 27, no. 2, pp. 73-75, 2019.

[58] L. Liu, "Observation on the curative effect of metoprolol combined with Qiliqiangxin capsule in the treatment of chronic heart failure," China Practical Medicine, vol. 14, no. 33, pp. 95-96, 2019.

[59] Y. Liu, "Observation on the effect of metoprolol combined with Qiliqiangxin capsule on chronic heart failure," Clinical Medicine, vol. 39, no. 12, pp. 116-117, 2019.

[60] W. He, "Evaluation of the curative effect of metoprolol combined with qiliqiangxin capsule in the treatment of chronic heart failure," Journal of Medical Aesthetics and Cosmetology, vol. 28, no. 5, p. 90, 2019.

[61] L. Wang, "Clinical observation of qiliqiangxin capsules combined with shakuba valsartan in the treatment of chronic heart failure," Chinese Journal of Clinical Rational Drug Use, vol. 12, no. 29, pp. 3-4, 2019.

[62] Y. Zhou, The Effects of Lvarabrine Combined with Qiliqiangxin Capsule on the Heart Rate Variability and Cardiac Structure and Function in Chronic Heart Failure Patients with Midrange Ejection Fraction, Southwest Medical University, Luzhou, China, 2019.

[63] X. Zhang, "Effects of sodium nitroprusside combined with qiliqiangxin capsule on chronic congestive heart failure," Chinese Journal of Heart and Heart Rhythm, vol. 7, no. 2, pp. 77-79, 2019. 
[64] S. Li, J. Xu, P. Chen et al., "Therapeutic effect of qiliqiangxin capsule combined with coenzyme Q10 on chronic heart failure and their influence on echocardiography, copotin and galectin-3 levels," Journal of Guangdong Medical University, vol. 38, no. 4, pp. 407-409, 2020.

[65] C. Guo, C. Fang, X. Fan, W. Jia, and W. Zhang, "Clinical study on qili qiangxin cadsules combined with enalapril maleate and folic acid tablets for chronic heart failure," Journal of New Chinese Medicine, vol. 52, no. 4, pp. 30-33, 2020.

[66] C. Su, J. Xu, P. Chen, D. Lv, S. Li, and Y. Tang, "Clinical effect of Qiliqiangxin capsules combined with sacubitril valsartan sodium tablets in the treatment of chronic heart failure and their influence on the somelysin-2 level," Journal of Guangdong Medical College, vol. 38, no. 1, pp. 37-40, 2020.

[67] J. Xu, "Clinical study of qiliqiangxin capsule combined with sacubitril and valsartan sodium tablets in the treatment of chronic heart failure," Journal of Practical Traditional Chinese Medicine, vol. 36, no. 4, pp. 507-509, 2020.

[68] C. Li, M. Zhang, H. Zhao et al., "Clinical observation on the curative effect of sakubatrovalsartan combined with qiliqiangxin capsule in the treatment of patients with chronic cardiac failure," Chinese Journal of Difficult and Complicated Cases, vol. 19, no. 7, pp. 667-671, 2020.

[69] C. Liu, "Efficacy evaluation of qiliqiangxin capsule combined with L-carnitine in the treatment of chronic heart failure," Capital Food Medicine, vol. 27, no. 14, p. 84, 2020.

[70] L. Wang, "Clinical observation of qili qiangxin capsule combined with L-carnitine in treating chronic heart failure," Special Health, vol. 30, pp. 98-99, 2020.

[71] X. Ding, T. Zhang, and Q. Yang, "Clinical observation on treatment of chronic congestive heart failure with sodium nitroprusside and qiliqiangxin capsule," Medical Information, vol. 24, no. 8, pp. 4933-4934, 2011.

[72] A. Pierobon, N. Granata, V. Torlaschi et al., "Psychomotor speed as a predictor of functional status in older chronic heart failure (CHF) patients attending cardiac rehabilitation," PLoS One, vol. 15, no. 7, Article ID e0235570, 2020.

[73] Z. Qing-Chun, W. Zhen-Li, H. Yu-Li et al., "Effects of qili qiangxin capsule on renal aquaporin-2 expression in rats with chronic heart failure," European Heart Journal Supplements, vol. 17, no. 3, 2015.

[74] H. Xiao, Y. Song, Y. Li, Y.-H. Liao, and J. Chen, "Qiliqiangxin regulates the balance between tumor necrosis factor- $\alpha$ and interleukin-10 and improves cardiac function in rats with myocardial infarction," Cellular Immunology, vol. 260, no. 1, pp. 51-55, 2009.

[75] Y. Gao, C. Xing, W. Hao et al., "The impact of sacrubitril/ valsartan on clinical treatment and hs-cTnT and NT-ProBNP serum levels and the left ventricular function in patients with chronic heart failure," International Heart Journal, vol. 61, no. 1, pp. 1-6, 2020.

[76] F. Triposkiadis, A. Xanthopoulos, J. Parissis, J. Butler, and D. Farmakis, "Pathogenesis of chronic heart failure: cardiovascular aging, risk factors, comorbidities, and disease modifiers," Heart Failure Reviews, 2020. 